\title{
PARITY AND STREETT GAMES WITH COSTS*
}

\author{
NATHANAËL FIJALKOW ${ }^{a}$ AND MARTIN ZIMMERMANN $^{b}$ \\ ${ }^{a}$ LIAFA, Université Paris 7 and Institute of Informatics, University of Warsaw \\ e-mail address: nath@liafa.univ-paris-diderot.fr \\ ${ }^{b}$ Reactive Systems Group, Saarland University \\ e-mail address: zimmermann@react.uni-saarland.de
}

\begin{abstract}
We consider two-player games played on finite graphs equipped with costs on edges and introduce two winning conditions, cost-parity and cost-Streett, which require bounds on the cost between requests and their responses. Both conditions generalize the corresponding classical omega-regular conditions and the corresponding finitary conditions.

For parity games with costs we show that the first player has positional winning strategies and that determining the winner lies in NP and coNP. For Streett games with costs we show that the first player has finite-state winning strategies and that determining the winner is EXPTIME-complete. The second player might need infinite memory in both games. Both types of games with costs can be solved by solving linearly many instances of their classical variants.
\end{abstract}

\section{INTRODUCTION}

In recent years, boundedness problems arose in topics pertaining to automata and logics leading to the development of novel models and techniques to tackle these problems. Although in general undecidable, many boundedness problems for automata turn out to be decidable if the acceptance condition can refer to boundedness properties of variables, but the transitions cannot access variable values. A great achievement was made by Hashiguchi 21] who proved decidability of the star-height problem by reducing it to a boundedness problem for a certain type of finite automaton and by then solving this problem. This led the path to recent developments towards a general theory of bounds in automata and logics, comprising automata and logics with bounds [2, 4, satisfiability algorithms for these logics [3, 5, 32, and regular cost-functions [14.

In this work, we consider boundedness problems in turn-based two-player graph games of infinite duration. We introduce cost-parity and cost-Streett conditions which generalize

2012 ACM CCS: [Software and its engineering]: Software organization and properties-Software functional properties-Formal methods; [Theory of computation]: Formal languages and automata theory-Automata extensions-Quantitative automata .

Key words and phrases: Parity Games, Streett Games, Costs, Half-positional Determinacy.

* A preliminary version of this work appeared in FSTTCS 2012 under the name "Cost-parity and CostStreett Games" 20. The research leading to these results has received funding from the European Union's Seventh Framework Programme (FP7/2007-2013) under grant agreements 259454 (GALE) and 239850 (SOSNA). 
the (classical) $\omega$-regular parity- respectively Streett condition, as well as the finitary parityrespectively finitary Streett condition [11].

A game with cost-parity condition is played on an arena whose vertices are colored by natural numbers, and where traversing an edge incurs a non-negative cost. The cost of a play (prefix) is the sum of the costs of the edges along the play (prefix). Player 0 wins a play if there is a bound $b$ such that all but finitely many odd colors seen along the play (which we think of as requests) are followed by a larger even color (which we think of as responses) that is reached with cost at most $b$. If all edges have cost zero, then this reduces to the parity condition: all but finitely many odd colors are followed by a larger even color. If all edges have positive cost, then this reduces to finitary parity conditions: there is a bound $b$ such that all but finitely many odd colors are followed by a larger even color within $b$ steps. The definition of the cost-Streett condition goes along the same lines, but the requests and responses are independent and not hierarchically ordered as in parity conditions.

The cost of traversing an edge can be used to model the consumption of a resource. Thus, if Player 0 wins a play she can achieve her goal along an infinite run with bounded resources. On the other hand, Player 1's objective is to exhaust the resource, no matter how big the capacity is. Note that this is not an $\omega$-regular property, which is witnessed by the fact that Player 1 needs infinite memory to win such games.

Since the term "cost-parity games" has been used before [14, 15, 32], we refer to games with cost-parity conditions as parity games with costs. The first difference between costparity games and parity games with costs is the bound quantification: in cost-parity games the counter values are required to be uniformly bounded over all paths, whereas in parity games with costs the bound can depend on the path. However, as shown in [10] in a more general context, the two formulations are equivalent over finite arenas. The actually relevant difference between cost-parity games and parity games with costs is in the intent: costparity games were introduced to solve the domination problem for regular cost-functions over finite trees [15]. Hence cost-parity games are very general: their winning conditions are conjunctions of a parity condition and of boundedness requirements on counters, allowing the counters and the parity condition to evolve independently. In contrast to this work, we are interested in efficient algorithms to solve games. Hence, in parity games with costs we restrict the use of counters, which are only used to give a quantitative measure of the satisfaction of the parity condition. This gives rise to a better-behaved winning condition, for which we provide a finer analysis of the complexity of solving the games and of the memory requirements.

We show that parity games with costs enjoy two nice properties of parity and finitary parity games: Player 0 has memoryless winning strategies and determining the winner lies in NP $\cap$ coNP 1. Furthermore, we show that solving parity games with costs can be algorithmically reduced to solving parity games, which allows to solve these games almost as efficiently as parity games. We then consider Streett games with costs and prove that Player 0 has finite-state winning strategies, and that determining the winner is EXPTIMEcomplete.

This unifies the previous results about finitary parity and Streett games and the results about their classical variants, in the following sense. For both parity and Streett, recall that the games with costs generalize both the classical and the finitary variants, hence solving them is at least as hard as solving these two subcases. Our results show that it

\footnotetext{
${ }^{1}$ This was recently improved to $\mathbf{U P} \cap \mathbf{c o U P}[27$.
} 
does not get worse: solving games with costs is not harder than solving the corresponding classical and finitary games. Indeed, solving finitary parity games can be carried out in polynomial time [11, while no polynomial-time algorithm for parity games is yet known, and the decision problem for parity games is in NP $\cap$ coNP. The situation is reversed for Streett games, since solving them is coNP-complete [18] while solving finitary Streett games is EXPTIME-complete. The latter result is shown in unpublished work by Chatterjee, Henzinger, and Horn: by slightly modifying the proof of EXPTIME-hardness of solving request-response games presented in 12 they prove EXPTIME-hardness of solving finitary Streett games.

To obtain our results, we present an algorithm to solve parity games with costs that iteratively computes the winning region of Player 0 employing an algorithm to solve parity games. This "reduction" to parity games also yields finite-state winning strategies for Player 0 in parity games with costs. However, this can be improved: by exploiting the intrinsic structure of the memory introduced in the reduction, we are able to prove the existence of positional winning strategies for Player 0 . We also give a second proof of this result: we show how to transform an arbitrary finite-state winning strategy into a positional one. This construction relies on so-called scoring functions (which are reminiscent of the simulation of alternating tree-automata by non-deterministic automata presented in [29] and of scoring functions for Muller games [26]) and presents a general framework to turn finitestate strategies into positional ones, which we believe to be applicable in other situations as well. Finally, we present an algorithm that solves Streett games with costs by solving Streett games. Here, we show the existence of finite-state winning strategies for Player 0 in Streett games with costs.

Adding quantitative requirements to qualitative winning conditions has been an active field of research during the last decade: much attention is being paid to not just synthesize some winning strategy, but to find an optimal one according to a certain quality measure, e.g., the use of mean-payoff objectives and weighted automata to model quantitative aspects in the winning condition [1, 8, 13]. For request-response games and their extensions, waiting times between requests and their responses are used to measure the quality of a strategy and it was shown how to compute optimal (w.r.t. the limit superior of the mean waiting time) winning strategies [22, 34]. However, the optimal finite-state strategies that are obtained are exponentially larger than the ones computed by the classical algorithm.

Finally, there has been a lot of interest in so-called energy games, whose winning conditions ask for the existence of an initial amount of energy such that a positive energy level is maintained throughout the play. Solving energy games with multiple resources is in general intractable [19] while so-called consumption games, a subclass of energy games, are shown to be tractable in [6]. Furthermore, energy parity games, whose winning conditions are a conjunction of a (single resource) energy and a parity condition, can be solved in $\mathbf{N P} \cap \mathbf{c o N P}$ and one player (the spoiling one) has positional winning strategies while the other one needs exponential memory [9]. The memory requirements show that energy parity games are incomparable to parity games with costs, since the second player needs infinite memory in the latter one. This also implies that there are no continuous reductions between these games via finite memory structures (see the next section for a formal definition of such reductions).

The paper is organized as follows. In Section 2, we define the necessary material related to games and introduce cost-parity and cost-Streett conditions, as well as their bounded variants, which are used to solve games with costs. In Section 3, we study bounded parity 
games with costs, providing an algorithm to solve them and tight memory requirements for winning strategies. In Section 4, we show how to reduce the problem of solving parity games with costs to the problem of solving bounded parity games with costs. In Section 5 , we give a different proof of the existence of positional strategies for (bounded) parity games with costs, via scoring-functions. In Section 6, we study Streett games with costs.

\section{Definitions}

We denote the non-negative integers by $\mathbb{N}$ and define $[n]=\{0,1, \ldots, n-1\}$ for every $n \geq 1$.

An arena $\mathcal{A}=\left(V, V_{0}, V_{1}, E\right)$ consists of a finite, directed graph $(V, E)$ and a partition $\left\{V_{0}, V_{1}\right\}$ of $V$ into the positions of Player 0 (drawn as circles) and the positions of Player 1 (drawn as rectangles). A play in $\mathcal{A}$ starting in $v \in V$ is an infinite path $\rho=$ $\rho_{0} \rho_{1} \rho_{2} \cdots$ through $(V, E)$ such that $\rho_{0}=v$. To avoid the nuisance of dealing with finite plays, we assume every vertex to have an outgoing edge.

A game $\mathcal{G}=(\mathcal{A}$, Win $)$ consists of an arena $\mathcal{A}$ and a set Win $\subseteq V^{\omega}$ of winning plays for Player 0 . The set of winning plays for Player 1 is $V^{\omega} \backslash$ Win. We say that Win is prefixindependent, if $\rho \in$ Win if and only if $w \rho \in$ Win for every play prefix $w$ and every infinite play $\rho$.

A strategy for Player $i$ is a mapping $\sigma: V^{*} V_{i} \rightarrow V$ such that $(v, \sigma(w v)) \in E$ for all $w v \in V^{*} V_{i}$. We say that $\sigma$ is positional if $\sigma(w v)=\sigma(v)$ for every $w v \in V^{*} V_{i}$. We often view positional strategies as a mapping $\sigma: V_{i} \rightarrow V$. A play $\rho_{0} \rho_{1} \rho_{2} \ldots$ is consistent with $\sigma$ if $\rho_{n+1}=\sigma\left(\rho_{0} \cdots \rho_{n}\right)$ for every $n$ with $\rho_{n} \in V_{i}$. A strategy $\sigma$ for Player $i$ is a winning strategy from a set of vertices $W \subseteq V$ if every play that starts in some $v \in W$ and is consistent with $\sigma$ is won by Player $i$. The winning region $W_{i}(\mathcal{G})$ of Player $i$ in $\mathcal{G}$ is the set of vertices from which Player $i$ has a winning strategy. We say that a strategy is uniform, if it is winning from all $v \in W_{i}(\mathcal{G})$. We always have $W_{0}(\mathcal{G}) \cap W_{1}(\mathcal{G})=\emptyset$. On the other hand, if $W_{0}(\mathcal{G}) \cup W_{1}(\mathcal{G})=V$, then we say that $\mathcal{G}$ is determined. All games we consider in this work are determined. Solving a game amounts to determining its winning regions and winning strategies.

A memory structure $\mathcal{M}=\left(M\right.$, Init, Upd) for an arena $\left(V, V_{0}, V_{1}, E\right)$ consists of a finite set $M$ of memory states, an initialization function Init: $V \rightarrow M$, and an update function Upd: $M \times V \rightarrow M$. The update function can be extended to $\mathrm{Upd}^{+}: V^{+} \rightarrow M$ in the usual way: $\operatorname{Upd}^{+}\left(\rho_{0}\right)=\operatorname{Init}\left(\rho_{0}\right)$ and $\operatorname{Upd}^{+}\left(\rho_{0} \cdots \rho_{n} \rho_{n+1}\right)=\operatorname{Upd}\left(\operatorname{Upd}^{+}\left(\rho_{0} \cdots \rho_{n}\right), \rho_{n+1}\right)$. A next-move function (for Player $i)$ Nxt: $V_{i} \times M \rightarrow V$ has to satisfy $(v, \operatorname{Nxt}(v, m)) \in E$ for all $v \in V_{i}$ and all $m \in M$. It induces a strategy $\sigma$ for Player $i$ with memory $\mathcal{M}$ via $\sigma\left(\rho_{0} \cdots \rho_{n}\right)=\operatorname{Nxt}\left(\rho_{n}, \operatorname{Upd}^{+}\left(\rho_{0} \cdots \rho_{n}\right)\right)$. A strategy is called finite-state if it can be implemented by a memory structure.

An arena $\mathcal{A}=\left(V, V_{0}, V_{1}, E\right)$ and a memory structure $\mathcal{M}=(M$, Init, Upd $)$ for $\mathcal{A}$ induce the expanded arena $\mathcal{A} \times \mathcal{M}=\left(V \times M, V_{0} \times M, V_{1} \times M, E^{\prime}\right)$ where $\left((v, m),\left(v^{\prime}, m^{\prime}\right)\right) \in E^{\prime}$ if and only if $\left(v, v^{\prime}\right) \in E$ and $\operatorname{Upd}\left(m, v^{\prime}\right)=m^{\prime}$. Every play $\rho$ in $\mathcal{A}$ has a unique extended play $\rho^{\prime}=\left(\rho_{0}, m_{0}\right)\left(\rho_{1}, m_{1}\right)\left(\rho_{2}, m_{2}\right) \ldots$ in $\mathcal{A} \times \mathcal{M}$ defined by $m_{0}=\operatorname{Init}\left(\rho_{0}\right)$ and $m_{n+1}=$ $\operatorname{Upd}\left(m_{n}, \rho_{n+1}\right)$, i.e., $m_{n}=\operatorname{Upd}^{+}\left(\rho_{0} \cdots \rho_{n}\right)$.

A game $\mathcal{G}=(\mathcal{A}$, Win $)$ is reducible to $\mathcal{G}^{\prime}=\left(\mathcal{A}^{\prime}\right.$, Win') via $\mathcal{M}$, written $\mathcal{G} \leq_{\mathcal{M}} \mathcal{G}^{\prime}$, if $\mathcal{A}^{\prime}=\mathcal{A} \times \mathcal{M}$ and every play $\rho$ in $\mathcal{G}$ is won by the player who wins the extended play $\rho^{\prime}$ in $\mathcal{G}^{\prime}$, i.e., $\rho \in \mathrm{Win}$ if and only if $\rho^{\prime} \in \mathrm{Win}^{\prime}$. 
Lemma 2.1. Let $\mathcal{G}$ be a game with vertex set $V$ and $W \subseteq V$. If $\mathcal{G} \leq_{\mathcal{M}} \mathcal{G}^{\prime}$ and Player $i$ has a positional winning strategy for $\mathcal{G}^{\prime}$ from $\{(v, \operatorname{Init}(v)) \mid v \in W\}$, then she has a finite-state winning strategy for $\mathcal{G}$ from $W$ which is implemented by $\mathcal{M}$.

Especially, if Player $i$ has a uniform positional winning strategy for $\mathcal{G}^{\prime}$, then she has a uniform finite-state winning strategy for $\mathcal{G}$ that is implemented by $\mathcal{M}$.

Let $\mathcal{A}=\left(V, V_{0}, V_{1}, E\right)$ and $i \in\{0,1\}$. The $i$-attractor of $F \subseteq V$ in $\mathcal{A}$, denoted by $\operatorname{Attr}_{i}^{\mathcal{A}}(F)$, is defined by $\operatorname{Attr}_{i}^{\mathcal{A}}(F)=\bigcup_{j=0}^{|V|} A_{j}$, where $A_{0}=F$ and

$$
\begin{aligned}
A_{j+1}=A_{j} & \cup\left\{v \in V_{i} \mid \exists v^{\prime} \in A_{j} \text { such that }\left(v, v^{\prime}\right) \in E\right\} \\
\cup\{v & \left.\in V_{1-i} \mid \forall v^{\prime},\left(v, v^{\prime}\right) \in E \text { implies } v^{\prime} \in A_{j}\right\} .
\end{aligned}
$$

Player $i$ has a positional strategy such that every play that $\operatorname{starts} \operatorname{in} \operatorname{Attr}_{i}^{\mathcal{A}}(F)$ and is consistent with the strategy visits $F$. Such strategies are called attractor strategies.

A trap for Player $i$ is a set $X$ of vertices such that the successors of every vertex in $X \cap V_{i}$ are again in $X$ and every vertex in $X \cap V_{1-i}$ has a successor in $X$. Player $1-i$ has a positional strategy such that every play that starts in a trap $X$ and is consistent with the

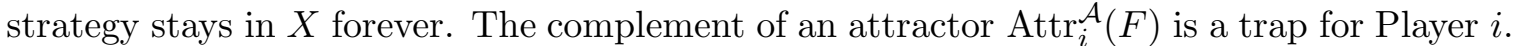
Furthermore, removing an attractor from an arena never introduces terminal vertices.

The following observation will be useful later: if the set of winning plays Win in $\mathcal{G}$ is prefix-independent, then we have $W_{i}(\mathcal{G})=\operatorname{Attr}_{i}^{\mathcal{A}}\left(W_{i}(\mathcal{G})\right)$ and $W_{i}(\mathcal{G})$ is a trap for Player $1-i$. Furthermore, no play consistent with a winning strategy for Player $i$ will ever leave $W_{i}(\mathcal{G})$.

2.1. Winning Conditions. In this subsection, we present the winning conditions we consider in this paper. Fix an arena $\mathcal{A}$ with set of edges $E$. A cost function for $\mathcal{A}$ is an edge-labelling Cst: $E \rightarrow\{\varepsilon, i\}$. Edges labelled with $i$ are called increment-edges while edges labelled by $\varepsilon$ are called $\varepsilon$-edges accordingly. We extend the edge-labelling to a cost function over plays obtained by counting the number of increment-edges traversed during the play, i.e., $\operatorname{Cst}(\rho) \in \mathbb{N} \cup\{\infty\}$. The cost of a play prefix is defined analogously.

Note that our definition of a cost function only allows cost zero or one on an edge. Alternatively, one could allow arbitrary costs in $\mathbb{N}$. This would not change our results, as we are interested in boundedness questions only. For the sake of simplicity, we refrain from using arbitrary costs in $\mathbb{N}$ and use abstract costs $\varepsilon$ and $i$ instead.

2.1.1. Cost-Parity Conditions. Let $\mathcal{A}=\left(V, V_{0}, V_{1}, E\right)$ be an arena and let $\Omega: V \rightarrow \mathbb{N}$ be a coloring of its vertices by natural numbers. In all games we are about to define in this subsection, we interpret the occurrence of a color as request, which has to be answered by visiting a vertex of larger or equal even color at an equal or later position. By imposing conditions on the responses we obtain several different types of winning conditions. To simplify our notations, let $\operatorname{Ans}(c)=\left\{c^{\prime} \in \mathbb{N} \mid c^{\prime} \geq c\right.$ and $c^{\prime}$ is even $\}$ be the set of colors that answer a request of color $c$. Note that $\operatorname{Ans}(c) \subseteq \operatorname{Ans}\left(c^{\prime}\right)$ for $c \geq c^{\prime}$ and $c \in \operatorname{Ans}(c)$ if $c$ is even.

Fix a cost function Cst and consider a play $\rho=\rho_{0} \rho_{1} \rho_{2} \cdots$ and a position $k \in \mathbb{N}$. We define the cost-of-response at position $k$ of $\rho$ by

$$
\operatorname{Cor}_{\mathrm{Cst}}(\rho, k)=\min \left\{\operatorname{Cst}\left(\rho_{k} \cdots \rho_{k^{\prime}}\right) \mid k^{\prime} \geq k \text { and } \Omega\left(\rho_{k^{\prime}}\right) \in \operatorname{Ans}\left(\Omega\left(\rho_{k}\right)\right)\right\},
$$

where we use $\min \emptyset=\infty$, i.e., $\operatorname{Cor}_{\mathrm{Cst}}(\rho, k)$ is the cost of the infix of $\rho$ from position $k$ to its first answer, and $\infty$ if there is no answer. 
We say that a request at position $k$ is answered with cost $b$, if $\operatorname{Cor}_{\mathrm{Cst}}(\rho, k)=b$. Note that a request at a position $k$ with an even color is answered with cost zero. Furthermore, we say that a request at position $k$ is unanswered with cost $\infty$, if there is no position $k^{\prime} \geq k$ such that $\Omega\left(\rho_{k^{\prime}}\right) \in \operatorname{Ans}\left(\Omega\left(\rho_{k}\right)\right)$ and we have $\operatorname{Cst}\left(\rho_{k} \rho_{k+1} \cdots\right)=\infty$, i.e., there are infinitely many increment-edges after position $k$, but no answer. Note that there is a third alternative: a request can be unanswered with finite cost, i.e., in case it is not answered, but the play $\rho$ contains only finitely many increment-edges.

We begin defining winning conditions by introducing the parity condition, denoted by $\operatorname{Parity}(\Omega)$, which requires that all but finitely many requests are answered. Equivalently, $\rho \in \operatorname{Parity}(\Omega)$ if and only if the maximal color that occurs infinitely often in $\rho$ is even. Both players have uniform positional winning strategies in parity games [17, 28] and their winning regions can be decided in NP $\cap$ coNP (and even in UP $\cap$ coUP2 [23]).

By bounding the costs between requests and their responses, we strengthen the parity condition and obtain the cost-parity and the bounded cost-parity condition. The former is defined as

$$
\operatorname{CostParity}(\Omega, \text { Cst })=\left\{\rho \in V^{\omega} \mid \limsup _{k \rightarrow \infty} \operatorname{Cor}_{\operatorname{Cst}}(\rho, k)<\infty\right\},
$$

i.e., $\rho$ satisfies the cost-parity condition, if there exists a bound $b \in \mathbb{N}$ such that all but finitely many requests are answered with cost less than $b$. The bounded cost-parity condition, denoted by BndCostParity $(\Omega$, Cst $)$, is again obtained by a strengthening:

$$
\begin{aligned}
\operatorname{BndCostParity}(\Omega, \text { Cst })=\left\{\rho \in V^{\omega}\right. & \mid \limsup _{k \rightarrow \infty} \operatorname{Cor}_{\text {Cst }}(\rho, k)<\infty \text { and } \\
& \text { no request in } \rho \text { is unanswered with cost } \infty\},
\end{aligned}
$$

i.e., $\rho$ satisfies the bounded cost-parity condition, if there exists a bound $b \in \mathbb{N}$ such that all but finitely many requests are answered with cost less than $b$, and there is no unanswered request of cost $\infty$. Note that this is not equivalent to requiring that there exists a bound $b^{\prime} \in$ $\mathbb{N}$ such that all requests are answered with cost less than $b^{\prime}$ (e.g., if there are unanswered requests in a play with finitely many increment-edges).

Remark 2.2. We have BndCostParity $(\Omega$, Cst $) \subseteq \operatorname{CostParity}(\Omega$, Cst $) \subseteq \operatorname{Parity}(\Omega)$ and $V^{*} \cdot \operatorname{BndCostParity}(\Omega$, Cst $)=\operatorname{CostParity}(\Omega$, Cst $)$. Furthermore, $\operatorname{CostParity}(\Omega$, Cst $)$ and Parity $(\Omega)$ are prefix-independent while $\operatorname{BndCostParity}(\Omega$, Cst $)$ is not.

A game $\mathcal{G}=(\mathcal{A}, \operatorname{CostParity}(\Omega$, Cst $))$ is called a parity game with costs, and a game with winning condition BndCostParity $(\Omega$, Cst $)$ is a bounded parity game with costs. Note that both cost-conditions defined here generalize the classical parity conditions as well as the finitary, respectively bounded, parity conditions of [11. Indeed, if $\mathcal{A}$ contains no incrementedges, then $\operatorname{CostParity}(\Omega$, Cst $)=\operatorname{BndCostParity}(\Omega$, Cst $)=\operatorname{Parity}(\Omega)$, the three conditions are equivalent. On the other hand, if $\mathcal{A}$ contains no $\varepsilon$-edges, then $\operatorname{CostParity}(\Omega, \mathrm{Cst})$ is equal to the finitary parity condition over $\Omega$ and $\operatorname{Bnd} \operatorname{CostParity}(\Omega$, Cst $)$ is equal to the bounded parity condition over $\Omega$. Hence, parity games with costs generalize both parity and finitary parity games. Similarly, bounded parity games with costs generalize both parity and bounded parity games.

Since (bounded) cost-parity conditions are Borel, we obtain determinacy of (bounded) parity games with costs via the Borel determinacy theorem.

\footnotetext{
${ }^{2} \mathrm{~A}$ problem is in $\mathbf{U P}$, if it can be decided by a non-deterministic polynomial-time Turing machine with at most one accepting run.
} 


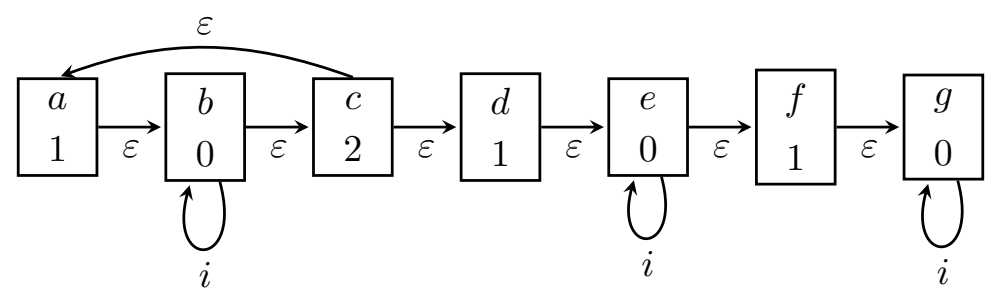

Figure 1: A (bounded) parity game with costs.

Lemma 2.3. (Bounded) parity games with costs are determined.

Proof. We show that both conditions are Borel. Then, the result follows from the Borel determinacy theorem [25].

We have

$$
\operatorname{CostParity}(\Omega, \text { Cst })=\bigcup_{\substack{n \in \mathbb{N} \\ b \in \mathbb{N}}} \bigcap_{k \geq n} \bigcup_{k^{\prime} \geq k} L_{b, k, k^{\prime}}
$$

where $L_{b, k, k^{\prime}}$ is the set of plays where the request at position $k$ is answered at position $k^{\prime}$ with cost at most $b$. Every $L_{b, k, k^{\prime}}$ is closed, hence CostParity ( $\Omega$, Cst) is in level $\Sigma_{4}$ of the Borel hierarchy ${ }^{3}$.

Furthermore, BndCostParity $(\Omega$, Cst) is equal to the intersection of $\operatorname{CostParity}(\Omega$, Cst) and the set

$$
\left\{\rho \in V^{\omega} \mid \text { no request in } \rho \text { is unanswered with cost } \infty\right\} .
$$

The latter set is recognizable by a parity automaton and therefore Borel. Hence, closure of Borel sets under intersection yields the desired result.

Example 2.4. Consider the parity game with costs depicted in Figure 1 where all vertices belong to $V_{1}$, and the label of a vertex denotes its name (in the upper part) and its color (in the lower part). Player 1 wins from $\{a, b, c\}$ by requesting color 1 at vertex $a$ infinitely often and staying at vertex $b$ longer and longer, but also visiting $c$ infinitely often (and thereby answering the request). Note that this strategy is not finite-state. Indeed, one can easily prove that Player 1 does not have a finite-state winning strategy for this game. Player 0 wins from every other vertex, since Player 1 can raise only finitely many requests from these vertices, albeit these requests are unanswered with cost $\infty$.

If we consider the game as a bounded parity game with costs, then Player 1 wins from every vertex but $g$ by moving to $g$ and then staying there ad infinitum. Every such play contains a request of color 1 that is unanswered with cost $\infty$. From $g$, Player 0 wins, since there is only one play starting from $g$, in which no request is ever raised.

\footnotetext{
${ }^{3}$ Note that this is not optimal: the cost-parity condition can be encoded in weak MSO with the unbounding quantifier. Such languages are boolean combinations of $\Sigma_{2}$ languages [3, which is optimal.
} 
2.1.2. Cost-Streett Conditions. Fix an arena $\mathcal{A}=\left(V, V_{0}, V_{1}, E\right)$. Let $\Gamma=\left(Q_{c}, P_{c}\right)_{c \in[d]}$ be a collection of $d$ (Streett) pairs of subsets of $V$, i.e., $Q_{c}, P_{c} \subseteq V$, and let $\overline{\mathrm{Cst}}=\left(\mathrm{Cst}_{c}\right)_{c \in[d]}$ be a collection of $d$ cost functions for $\mathcal{A}$. We think of visits to vertices in $Q_{c}$ as requests, visits to $P_{c}$ as responses, and measure the cost of these responses using Cst $_{c}$. Formally, for $c \in[d]$, a play $\rho=\rho_{0} \rho_{1} \rho_{2} \cdots$, and a position $k$ we define the cost-of-response by

$$
\operatorname{StCor}_{\mathrm{Cst}_{c}}(\rho, k)= \begin{cases}0 & \text { if } \rho_{k} \notin Q_{c}, \\ \min \left\{\operatorname{Cst}_{c}\left(\rho_{k} \cdots \rho_{k^{\prime}}\right) \mid k^{\prime} \geq k \text { and } \rho_{k^{\prime}} \in P_{c}\right\} & \text { if } \rho_{k} \in Q_{c},\end{cases}
$$

where we use $\min \emptyset=\infty$. We define $\operatorname{StCor}_{\overline{\mathrm{Cst}}}(\rho, k)=\max \left\{\operatorname{StCor}_{\mathrm{Cst}_{c}}(\rho, k) \mid c \in[d]\right\}$ and say that the requests at position $k$ are answered with cost $b$, if $\operatorname{StCor}_{\overline{C s t}}(\rho, k)=b$, and that the requests are unanswered with cost $\infty$, if $\operatorname{StCor}_{\overline{\mathrm{Cst}}}(\rho, k)=\infty$ and there are infinitely many increment-edges after position $k$ (w.r.t. some Cst $_{c}$ such that $\rho_{k} \in Q_{c}$ ). This rules out the case where we have $\operatorname{StCor}_{\overline{\mathrm{Cst}}}(\rho, k)=\infty$ due to a request at position $k$ that is not answered, but $\rho$ only traverses finitely many increment-edges.

We consider the following winning conditions. The (classical) Streett condition $\operatorname{Streett}(\Gamma)$ requires for every $c$ that $P_{c}$ is visited infinitely often if $Q_{c}$ is visited infinitely often, i.e., all but finitely many requests are answered.

Again, by requiring a bound on the costs between requests and responses, we strengthen the Streett condition: the cost-Streett condition

$$
\operatorname{CostStreett}(\Gamma, \overline{\mathrm{Cst}})=\left\{\rho \in V^{\omega} \mid \limsup _{k \rightarrow \infty} \operatorname{StCor}_{\overline{\mathrm{Cst}}}(\rho, k)<\infty\right\}
$$

requires the existence of a bound $b$ such that all but finitely many requests are answered with cost less than $b$. Finally, the bounded cost-Streett condition BndCostStreett $(\Gamma, \overline{\mathrm{Cst}})$ requires the existence of a bound $b$ such that all but finitely many requests are answered with cost less than $b$, and that there is no unanswered request of cost $\infty$. Formally, we define

$$
\begin{aligned}
& \operatorname{BndCostStreett}(\Gamma, \overline{\mathrm{Cst}})=\left\{\rho \in V^{\omega} \mid \limsup _{k \rightarrow \infty} \operatorname{StCor}_{\overline{\mathrm{Cst}}}(\rho, k)<\infty\right. \text { and } \\
& \text { no request in } \rho \text { is unanswered with cost } \infty\} \text {. }
\end{aligned}
$$

Remark 2.5. We have BndCostStreett $(\Gamma, \overline{\mathrm{Cst}}) \subseteq \operatorname{CostStreett}(\Gamma, \overline{\mathrm{Cst}}) \subseteq \operatorname{Streett}(\Gamma)$ and $V^{*} \cdot \operatorname{BndCostStreett}(\Gamma, \overline{\mathrm{Cst}})=\operatorname{CostStreett}(\Gamma, \overline{\mathrm{Cst}})$. Furthermore, CostStreett $(\Gamma, \mathrm{Cst})$ and Streett $(\Gamma)$ are prefix-independent while BndCostStreett $(\Gamma$, Cst) is not.

A game $(\mathcal{A}, \operatorname{CostStreett}(\Gamma, \overline{\mathrm{Cst}}))$ where $\Gamma$ and $\overline{\mathrm{Cst}}$ have the same size is called a Streett game with costs. As in the case for (bounded) cost-parity conditions, the winning conditions defined here generalize the classical Streett condition as well as the finitary, respectively, bounded Streett condition of [11. Indeed, if $\mathcal{A}$ contains no increment-edges, then the three

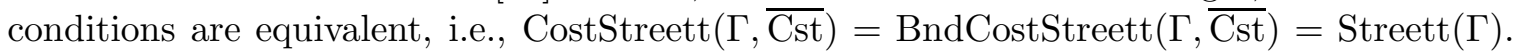
Similarly, if $\mathcal{A}$ contains no $\varepsilon$-edges, then $\operatorname{CostStreett}(\Gamma, \overline{\mathrm{Cst}})$ is equal to the finitary Streett condition over $\Gamma$ and BndCostParity $(\Gamma, \overline{\mathrm{Cst}})$ is equal to the bounded Streett condition over $\Gamma$. Hence, Streett games with costs generalize both Streett and finitary Streett games. Similarly, bounded Streett games with costs generalize both Streett and bounded Streett games. Furthermore, just as classical Streett games subsume parity games, Streett games with costs subsume parity games with costs, and bounded Streett games with costs subsume bounded parity games with costs. 


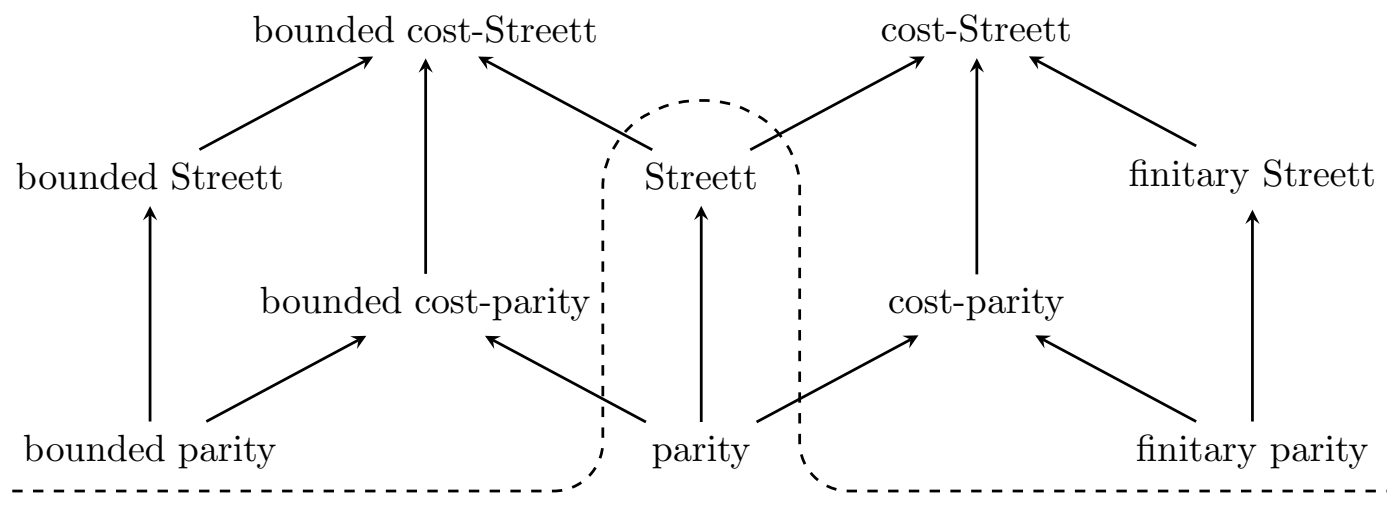

Figure 2: Expressiveness of winning conditions; those below the dashed line are $\omega$-regular.

Figure 2 shows the expressiveness of the winning conditions, e.g., the arrow from "bounded parity" to "bounded Streett" denotes that every bounded parity condition is also a bounded Streett condition.

Finally, we obtain determinacy via the Borel determinacy theorem.

Lemma 2.6. (Bounded) Streett games with costs are determined.

Proof. Analogously to the proof of Lemma 2.3.

\section{Bounded Parity Games with Costs}

In this section, we study bounded parity games with costs. We first show how to solve such games, and then consider the memory requirements for winning strategies for both players. Note that bounded parity games with costs are a generalization of parity games, hence the algorithm we present in the following subsection also has to solve parity games as a special case.

3.1. Solving Bounded Parity Games with Costs via $\omega$-regular Games. To solve bounded parity games with costs, we present a relaxation of the bounded cost-parity condition, called PCRR which is a boolean combination of a parity, a co-Büchi, and a requestresponse [33] condition (hence its name). This condition essentially replaces the bound $b$ on the cost between a request and its response by just requiring an answer to every request. Furthermore, for plays with finite cost it just requires the parity condition to be satisfied, just as the bounded cost-parity condition does. The PCRR-condition is $\omega$-regular, thus both players have finite-state winning strategies in games with PCRR winning conditions [7. Using the fact that a finite-state winning strategy for Player 0 answers every request within a fixed number of steps (and thereby also with bounded cost), we are able to show that these two games have the same winning regions. Finally, we show how to reduce the PCRR-condition to a parity condition. This completes our algorithm for solving bounded parity games with costs and also yields upper bounds on the memory requirements of both players in bounded parity games with costs.

Let $\mathcal{G}=(\mathcal{A}, \operatorname{Bnd} \operatorname{CostParity}(\Omega$, Cst $))$. First, we turn the cost function into a state property in order to be able to define cost-based winning conditions (which are sequences 
of vertices not edges): in the following, we assume that no vertex of $\mathcal{A}$ has both incoming increment- and $\varepsilon$-edges. This can be achieved by subdividing every increment-edge $e=$ $\left(v, v^{\prime}\right)$ : we add a new vertex $\operatorname{sub}(e)$ and replace $e$ by $(v, \operatorname{sub}(e))$ (which is an increment-edge) and by $\left(\operatorname{sub}(e), v^{\prime}\right)$ (which is an $\varepsilon$-edge). Now, only the newly added vertices have incoming increment-edges, but they do not have incoming $\varepsilon$-edges. Furthermore, it is easy to see that Player $i$ wins from a vertex in the original game if and only if she wins from this vertex in the modified game (where we color $\operatorname{sub}(e)$ by $\Omega\left(v^{\prime}\right)$ ). Finally, the modification does not change the memory requirements, e.g., if Player 0 has a positional winning strategy for the modified game, then also for the original game.

We say that a vertex is an increment-vertex, if it has an incoming increment-edge (which implies that all incoming edges are increment-edges). Let $I$ be the set of increment-vertices. Then, $\operatorname{coBüchi}(I)=\{\rho \mid \operatorname{Cst}(\rho)<\infty\}$ is the set of infinite plays having finite cost, i.e., those plays that visit only finitely many increment-vertices. Furthermore, by $\operatorname{RR}(\Omega)$ we denote the set of infinite plays in which every request is answered. We define

$$
\operatorname{PCRR}(\Omega, I)=(\operatorname{Parity}(\Omega) \cap \operatorname{coBüchi}(I)) \cup \operatorname{RR}(\Omega),
$$

which is $\omega$-regular, since it is a boolean combination of $\omega$-regular languages. Note that $\operatorname{PCRR}(\Omega, I)$ relaxes BndCostParity $(\Omega$, Cst $)$ by giving up the bound on the cost between requests and responses, in other words $\operatorname{PCRR}(\Omega, I) \supseteq \operatorname{BndCostParity}(\Omega$, Cst $)$.

Lemma 3.1. Let $\mathcal{G}=(\mathcal{A}, \operatorname{Bnd} \operatorname{CostParity}(\Omega, \operatorname{Cst}))$ and $\mathcal{G}^{\prime}=(\mathcal{A}, \operatorname{PCRR}(\Omega, I))$, where $I$ is defined as above. A finite-state winning strategy for Player $i$ in $\mathcal{G}^{\prime}$ from a set $W$ of vertices is also a winning strategy for Player $i$ in $\mathcal{G}$ from $W$.

Proof. The statement for $i=1$ follows from the inclusion $V^{\omega} \backslash \operatorname{PCRR}(\Omega, I) \subseteq V^{\omega} \backslash$ BndCostParity $(\Omega$, Cst).

Now, consider the case $i=0$ and let $\sigma$ be a finite-state winning strategy for Player 0 in $\mathcal{G}^{\prime}$ from $W$. We argue that $\sigma$ is also a winning strategy for Player 0 for $\mathcal{G}$ from $W$ : let $\rho$ be consistent with $\sigma$ and starting in $W$, which implies $\rho \in \operatorname{PCRR}(\Omega, I)$.

If $\rho$ satisfies Parity $(\Omega)$ and has only finitely many increments (say $b$ many), then all but finitely many requests are answered with cost less than $b+1$ and there is no unanswered request of cost $\infty$, i.e., $\rho \in \operatorname{Bnd} \operatorname{CostParity}(\Omega$, Cst $)$.

Otherwise, $\rho$ satisfies $\operatorname{RR}(\Omega)$, i.e., every request in $\rho$ is answered. We show that every request in $\rho$ is answered with cost at most $b=|V| \cdot|\sigma|$ (where $|\sigma|$ is the size of the memory structure implementing $\sigma$ ), which implies that $\rho \in \operatorname{BndCostParity}(\Omega$, Cst). Towards a contradiction, assume that there is a request that is answered with cost greater than $b$. Then, there are two positions between the request and its answer having the same vertex, an increment-edge in between them, and such that the memory structure implementing $\sigma$ assumes the same state at both positions. Hence, using this loop forever is also a play that is consistent with $\sigma$. However, this play contains an unanswered request of cost $\infty$ and therefore does not satisfy $\operatorname{PCRR}(\Omega, I)$. This yields the desired contradiction to the fact that $\sigma$ is a winning strategy.

Corollary 3.2. Let $\mathcal{G}$ and $\mathcal{G}^{\prime}$ as in Lemma 3.1. Then, $W_{i}(\mathcal{G})=W_{i}\left(\mathcal{G}^{\prime}\right)$ for $i \in\{0,1\}$.

We now show how to reduce $\mathcal{G}^{\prime}=(\mathcal{A}, \operatorname{PCRR}(\Omega, I))$ to a parity game only linearly larger than $\mathcal{G}$. Let $O$ be the set of odd colors in $\Omega(V)$. We define the memory structure $\mathcal{M}=$ 
(M, Init, Upd) with $M=O \cup\{\perp\}$,

$$
\operatorname{Init}(v)= \begin{cases}\Omega(v) & \text { if } \Omega(v) \text { odd } \\ \perp & \text { otherwise }\end{cases}
$$

$\operatorname{Upd}(\perp, v)=\operatorname{Init}(v)$, and

$$
\operatorname{Upd}(c, v)= \begin{cases}\max (\Omega(v), c) & \text { if } \Omega(v) \text { odd } \\ \perp & \text { if } \Omega(v) \in \operatorname{Ans}(c) \\ c & \text { otherwise. }\end{cases}
$$

Intuitively, $\operatorname{Upd}^{+}(w)$ is the largest unanswered request in $w$, and is $\perp$ if every request in $w$ is answered. Furthermore, let $\ell$ be an odd color that is larger than every color in $\Omega(V)$. Now, we define a coloring $\Omega_{\mathcal{M}}$ of the arena $\mathcal{A} \times \mathcal{M}$ via

$$
\Omega_{\mathcal{M}}(v, m)= \begin{cases}\ell+1 & \text { if } m=\perp, \\ \ell & \text { if } m \neq \perp \text { and } v \in I, \\ \Omega(v) & \text { otherwise. }\end{cases}
$$

So, having all requests answered (i.e., being in memory state $\perp$ ) is most desirable for Player 0 while visiting increment-vertices (i.e., vertices in $I$ ) while having an open request is most desirable for Player 1. If neither of these occurs infinitely often, then the old coloring $\Omega$ determines the winner (without taking the memory states into account).

Lemma 3.3. Let $\mathcal{G}^{\prime}=(\mathcal{A}, \operatorname{PCRR}(\Omega, I))$ and $\mathcal{G}^{\prime \prime}=\left(\mathcal{A} \times \mathcal{M}\right.$, Parity $\left.\left(\Omega_{\mathcal{M}}\right)\right)$. Then, $\mathcal{G}^{\prime} \leq_{\mathcal{M}} \mathcal{G}^{\prime \prime}$.

Proof. Let $\rho^{\prime}=v_{0} v_{1} v_{2} \cdots$ be a play in $\mathcal{A}$ and $\rho^{\prime \prime}=\left(v_{0}, m_{0}\right)\left(v_{1}, m_{1}\right)\left(v_{2}, m_{2}\right) \cdots$ be its extended play in $\mathcal{A} \times \mathcal{M}$. By construction, $m_{j}$ is the largest unanswered request in $v_{0} \cdots v_{j}$. We have to show that the same player wins both $\rho^{\prime}$ in $\mathcal{G}^{\prime}$ and $\rho^{\prime \prime}$ in $\mathcal{G}^{\prime \prime}$.

Assume $\rho^{\prime} \in \operatorname{PCRR}(\Omega, I)$. If $\rho^{\prime} \in \operatorname{RR}(\Omega)$, then every request is answered, i.e., $m_{j}$ is infinitely often equal to $\perp$. These vertices have the largest color in $\mathcal{G}^{\prime \prime}$, which is even. Hence, $\rho^{\prime \prime} \in \operatorname{Parity}\left(\Omega_{\mathcal{M}}\right)$. On the other hand, if $\rho^{\prime} \in \operatorname{Parity}(\Omega) \cap \operatorname{coBüchi}(I)$ but $\rho^{\prime} \notin \operatorname{RR}(\Omega)$, then $\rho^{\prime}$ and $\rho^{\prime \prime}$ each have a suffix (starting after the last occurrence of an increment-vertex or the last unanswered request, whichever comes last) such that these suffixes have the same sequence of colors. Hence, $\rho^{\prime \prime}$ satisfies $\operatorname{Parity}\left(\Omega_{\mathcal{M}}\right)$.

Conversely, assume $\rho^{\prime \prime} \in \operatorname{Parity}\left(\Omega_{\mathcal{M}}\right)$. If $\ell+1$ is the maximal color seen infinitely often, then $m_{j}$ is infinitely often equal to $\perp$, which implies that every request in $\rho^{\prime}$ is answered, i.e., $\rho^{\prime} \in \operatorname{RR}(\Omega) \subseteq \operatorname{PCRR}(\Omega, I)$. On the other hand, if the maximal color seen infinitely often is smaller than $\ell+1$ (but still even, since we assume Player 0 wins $\rho^{\prime \prime}$ ), then there are only finitely many increment-vertices in $\rho^{\prime}$ and the plays $\rho^{\prime}$ and $\rho^{\prime \prime}$ each have a suffix such that these suffixes have the same sequence of colors. Hence, $\rho^{\prime}$ satisfies Parity $(\Omega)$. Altogether, we have $\rho^{\prime} \in \operatorname{Parity}(\Omega) \cap \operatorname{coBüchi}(I) \subseteq \operatorname{PCRR}(\Omega, I)$.

Corollary 3.4. In bounded parity games with costs, both players have uniform finite-state winning strategies of size $d+1$, where $d$ is the number of odd colors in the game.

Proof. The reduction from games with winning condition $\operatorname{PCRR}(\Omega, I)$ to parity games yields uniform finite-state winning strategies of size $d+1$ for such games. Now apply Lemma 3.1. 
In the next subsection, we show this bound to be tight for Player 1 and show that Player 0 even has positional winning strategies.

The reduction from PCRR games to parity games and Lemma 3.1 show that solving a parity game suffices to solve a bounded parity game with costs and proves the following theorem. Here, $n$ is the number of vertices, $m$ is the number of edges, and $d$ is the number of colors in the game.

Theorem 3.5. Given an algorithm that solves parity games in time $T(n, m, d)$, there is an algorithm that solves bounded parity games with costs in time $O(T(d n, d m, d+2))$.

Furthermore, since solving parity games is in NP $\cap$ coNP and the blowup in our reduction is polynomial, we obtain the following remark (note that this was recently improved to $\mathbf{U P} \cap \mathbf{c o U P}[27])$.

Remark 3.6. The following problem is in NP $\cap$ coNP: given a bounded parity game with costs $\mathcal{G}, i \in\{0,1\}$, and a vertex $v$, is $v \in W_{i}(\mathcal{G})$ ?

Let us conclude by considering the special case of a bounded parity game with costs $\mathcal{G}$ in which every edge is an increment-edge, i.e., where $\mathcal{G}$ is a bounded parity game. These games, called "bounded parity games" in [11 can be solved in polynomial time. In this case, $\operatorname{PCRR}(\Omega$, Cst $)$ is equal to $\operatorname{RR}(\Omega)$, which is a request-response condition [33] where the sets of requests and responses form a hierarchy, induced by the order on the colors. It is easy to derive from the reduction to Büchi games [33] that such games can be solved in polynomial time. Hence, we have recovered the result of [11] on bounded parity games as a special case of our algorithm, although the running time of this algorithm is worse than the running time of the algorithm presented in [11].

3.2. Memory Requirements in Bounded Parity Games with Costs. In this subsection, we determine the exact memory requirements for both players in bounded parity games with costs. We begin by considering Player 0 and improve on Corollary 3.4 .

Lemma 3.7. In bounded parity games with costs, Player 0 has uniform positional winning strategies.

Proof. Due to Lemma 3.1, it suffices to prove the statement for games $\mathcal{G}^{\prime}=(\mathcal{A}, \operatorname{PCRR}(\Omega, I))$. Recall that we reduced such a game to a parity game $\mathcal{G}^{\prime \prime}=\left(\mathcal{A} \times \mathcal{M}\right.$, Parity $\left.\left(\Omega_{\mathcal{M}}\right)\right)$ using a memory structure $\mathcal{M}$ that keeps track of the largest open request. Specifically, Lemma 3.3 reads as follows: $v_{0} \in W_{0}\left(\mathcal{G}^{\prime}\right)$ if and only if $\left(v_{0}, \operatorname{Init}\left(v_{0}\right)\right) \in W_{0}\left(\mathcal{G}^{\prime \prime}\right)$.

We order $M=O \cup\{\perp\}$ with the natural order on integers for $O$, where $\perp$ is the minimal element. Player 0's winning region in $\mathcal{G}^{\prime \prime}$ is downwards-closed, i.e., $(v, m) \in W_{0}\left(\mathcal{G}^{\prime \prime}\right)$ and $m^{\prime}<m$ implies $\left(v, m^{\prime}\right) \in W_{0}\left(\mathcal{G}^{\prime \prime}\right)$, which can be shown by mimicking a winning strategy from $(v, m)$ to also win from $\left(v, m^{\prime}\right)$. Thus, for $v \in W_{0}\left(\mathcal{G}^{\prime}\right)$, we define

$$
\max (v)=\max \left\{m \in M \mid(v, m) \in W_{0}\left(\mathcal{G}^{\prime \prime}\right)\right\},
$$

which is well-defined as $(v, \operatorname{Init}(v)) \in W_{0}\left(\mathcal{G}^{\prime \prime}\right)$.

Now, let $\sigma^{\prime \prime}$ be a uniform positional winning strategy for Player 0 in the parity game $\mathcal{G}^{\prime \prime}$. We define a positional strategy $\sigma^{\prime}$ for $\mathcal{G}^{\prime}$ by using $\max (v)$, i.e., the worst memory state Player 0 could be in at vertex $v$ while still being able to win from there. Given a vertex $v \in$ $W_{0}\left(\mathcal{G}^{\prime}\right)$, let $\sigma^{\prime \prime}(v, \max (v))=\left(v^{\prime}, m^{\prime}\right)$. Using this, we define $\sigma^{\prime}(v)=v^{\prime}$. We show that $\sigma^{\prime}$ is a uniform winning strategy for Player 0 in $\mathcal{G}^{\prime}$. Consider a play $\rho^{\prime}=v_{0} v_{1} v_{2} \cdots$ starting in 
$v_{0} \in W_{0}\left(\mathcal{G}^{\prime}\right)$ consistent with $\sigma^{\prime}$, and $\rho^{\prime \prime}=\left(v_{0}, m_{0}\right)\left(v_{1}, m_{1}\right)\left(v_{2}, m_{2}\right) \cdots$ its extended play in $\mathcal{A} \times \mathcal{M}$. A straightforward induction shows that for every $j$, we have $\left(v_{j}, m_{j}\right) \in W_{0}\left(\mathcal{G}^{\prime \prime}\right)$, so $\max \left(v_{j}\right) \geq m_{j}$. We have to show $\rho^{\prime} \in \operatorname{PCRR}(\Omega, I)$.

By Lemma 3.3, $\rho^{\prime} \in \operatorname{PCRR}(\Omega, I)$ if and only if $\rho^{\prime \prime} \in \operatorname{Parity}\left(\Omega_{\mathcal{M}}\right)$. Assume towards a contradiction that the maximal color seen infinitely often in $\rho^{\prime \prime}$ is odd. This implies that the memory state $\perp$ appears finitely often, so after a position, say $n$, all memory states are different from $\perp$. Furthermore, from position $n$, we additionally have $\max \left(v_{j}\right) \leq \max \left(v_{j+1}\right)$; this follows from the observation that if $\operatorname{Upd}(c, v) \neq \perp$, then $c \leq \operatorname{Upd}(c, v)$. Consider $\rho^{*}=$ $\left(v_{n}, \max \left(v_{n}\right)\right)\left(v_{n+1}, \max \left(v_{n+1}\right)\right)\left(v_{n+2}, \max \left(v_{n+2}\right)\right) \cdots$. Since the sequence $\left(\max \left(v_{j}\right)\right)_{j \geq n}$ is non-decreasing, it is ultimately constant, say from position $n^{\prime} \geq n$. The suffix starting from $n^{\prime}$ is consistent with $\sigma^{\prime \prime}$ and starts in $W_{0}\left(\mathcal{G}^{\prime \prime}\right)$, so it satisfies $\operatorname{Parity}\left(\Omega_{\mathcal{M}}\right)$ since $\sigma^{\prime \prime}$ is a winning strategy. Consequently, $\rho^{*}$ contains finitely many increment-vertices, so $\rho^{\prime \prime}$ as well. After the last increment-vertex, $\rho^{*}$ and $\rho^{\prime \prime}$ have the same colors, but $\rho^{\prime \prime}$ does not satisfy $\operatorname{Parity}\left(\Omega_{\mathcal{M}}\right)$, a contradiction.

To conclude this subsection, we prove that the upper bound $d+1$ on the memory requirements of Player 1 proved in Corollary 3.4 is tight.

Lemma 3.8. For every $d \geq 1$, there is a bounded parity game with costs $\mathcal{G}_{d}$ such that

- the arena of $\mathcal{G}_{d}$ is of linear size in $d$ and there are $d$ odd colors in $\mathcal{G}_{d}$,

- Player 1 has a uniform finite-state winning strategy for $\mathcal{G}_{d}$ from every vertex, which is implemented with $d+1$ memory states, but

- there is a vertex from which Player 1 has no winning strategy that is implemented with less than $d+1$ memory states.

Proof. We begin by describing the game by an example: Figure 3 depicts the game $\mathcal{G}_{4}$, where the numbers in the vertices denote their colors. Since each edge is an increment-edge, we do not label them as such in the picture. The arena consists of a hub vertex colored by 0 and four disjoint blades, which are identified by the odd color of their outermost vertex, i.e., by the colors $1,3,5$ and 7 (which is $2 \cdot 4-1$ ). From the hub, Player 0 can enter the blade for an odd color $c$ at a vertex of color $c-1$ (which is even) which has a self-loop and an edge to a vertex of color 8 (which answers every request in the game). This vertex has only one outgoing edge to a vertex of color $c$ (this is the identifying color). Again, this vertex has only one successor, the hub. In general, the arena of $\mathcal{G}_{d}$ has $d$ blades, one for each color in $\{1,3, \ldots, 2 d-1\}$, the hub has color 0 , and the second vertex in each blade has color $2 d$ and thereby answers every request. Furthermore, every edge is an increment-edge.

At the hub, Player 0 picks a blade (say of color $c$ ) and then Player 1 decides whether to use the self-loop or to return to the hub. Note that Player 0 loses, if she enters the blade of color $c$ while there is an open request of some color $c^{\prime}>c$, since Player 1 can use the self-loop of the blade and thereby prevent an answer to the request $c^{\prime}$. On the other hand, if Player 1 decides to leave the blade, all requests are answered and then color $c$ is requested. Note that this request is never answered to by moving to the hub.

First, we show that Player 1 has a uniform finite-state winning strategy from every vertex that is implementable with $d+1$ memory states. The memory structure keeps track of the largest open request, i.e., we use states $1,3, \ldots, 2 d-1$ and an additional state $\perp$ that is reached, if there is no open request. Now, assume the current memory state is $m$ and the play is in a vertex of Player 1, which is uniquely identified by its even color $c$. If $m=\perp$, then Player 1 moves to the (unique) successor of color $2 d$. Now, assume $m \neq \perp$, i.e., $m$ is 


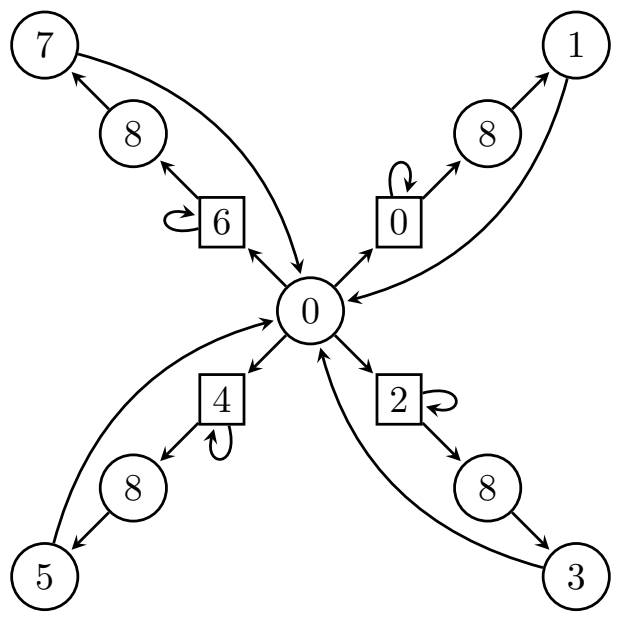

Figure 3: The bounded parity game with costs $\mathcal{G}_{4}$ (every edge is an increment-edge).

some odd color. If $m<c$, then Player 1 again leaves $c$ by moving to the unique successor of color $2 d$. If $m>c$, then Player 1 uses the self-loop forever.

Now, consider a play that is consistent with this strategy. If the current memory state is $\perp$, then a request is raised within the next three moves and the play returns to the hub, which implies that the memory is updated to some odd color $m$. From there, Player 0 has to move to some blade, say for color $c$ (which is odd). If $m>c$, then Player 1 uses the self-loop at the vertex of color $c-1$ forever. The resulting play is winning for him, since the request of $c$ is unanswered with cost $\infty$. On the other hand, if $m<c$, then Player 1 moves to the vertex of color $c$ and then back to the hub. While doing this, the memory is updated to a larger state, namely $c$. Hence, the memory states along a play consistent with the strategy described above are increasing, which means that at some point Player 0 has to enter a blade for color $c<m$, where $m$ is the current memory state, i.e., also an open request. Then, Player 1 will win by using the self-loop of this blade. Hence, the strategy described above is a winning strategy from every vertex and is implemented using $d+1$ memory states.

It remains to show that the upper bound $d+1$ is tight. To this end, consider a finite-state strategy $\tau$ for Player 1 that is winning from the hub, say $\tau$ is implemented by ( $M$, Init, Upd). We show that $M$ contains at least $d+1$ memory states. To this end, we define a sequence $m_{0}, m_{1}, \ldots, m_{d}$ of $d+1$ memory states, as follows. Define $m_{0}=\operatorname{Init}(v)$, where $v$ is the hub. Now, Consider the play where Player 0 moves from the hub to the blade with color 1 . Since $\tau$ is a winning strategy, Player 1 will use the self-loop of this blade only finitely often, i.e., the hub is reached again. We denote this play prefix by $w_{1}$ (which is consistent with $\tau)$ and define $m_{1}=\operatorname{Upd}^{+}\left(w_{1}\right)$. Consider now the play where after $w_{1}$, Player 0 moves to the blade with color 3 . Again, Player 1 will use the self-loop only finitely often and the hub is reached again. We denote the prolongation of $w_{1}$ through this blade by $w_{2}$ and define $m_{2}=\operatorname{Upd}^{+}\left(w_{1} w_{2}\right)$. This process is continued for each blade in ascending order. Since Player 1 has to leave each blade we obtain a sequence $m_{0}, m_{1}, \ldots, m_{d}$ of memory states assumed at the visits of the hub and a play prefix $w_{1} w_{2} \cdots w_{d}$ that is consistent with $\tau$, 
starts in the hub, and satisfies $\operatorname{Upd}^{+}\left(w_{1} w_{2} \cdots w_{j}\right)=m_{j}$ for every $j \geq 1$. Furthermore, each $w_{1} w_{2} \cdots w_{j}$ ends in the hub.

We argue that the states $m_{0}, m_{1}, \ldots, m_{d}$ are pairwise distinct. Assume towards contradiction there are $j<j^{\prime} \leq d$ such that $m_{j}=m_{j^{\prime}}$. Then, the play $\rho=w_{1} \cdots w_{j} \cdot\left(w_{j+1} \cdots w_{j^{\prime}}\right)^{\omega}$ is consistent with $\tau$. However, the maximal color seen infinitely often during $\rho$ is $2 d$ (which answers every request), and there is a uniform bound on the distance between the occurrences of $2 d$. Hence, the play is winning for Player 0 in $\mathcal{G}_{d}$, contradicting the fact that $\tau$ is a winning strategy for Player 1 . Hence, the states $m_{j}$ are indeed pairwise distinct. Thus, every winning strategy has at least $d+1$ memory states.

Recall that every edge in $\mathcal{G}_{d}$ is an increment-edge, i.e., $\mathcal{G}_{d}$ is a bounded parity game. In [11 an upper bound of two on the memory requirements of Player 1 is claimed for bounded parity games. The games presented here refute this claim: there is no constant bound on the memory needed for Player 1 in bounded parity games.

\section{Solving Parity Games with Costs via Bounded Parity Games with Costs}

In this section, we show that being able to solve bounded parity games with costs suffices to solve parity games with costs. Our algorithm is based on the following lemma which formalizes this claim by relating the winning regions of Player 0 in a parity game with costs and the bounded parity game with costs in the same arena. The algorithm presented here is equal to the one presented to solve finitary parity games by solving bounded parity games [11. However, our correctness proof is more general, since it has to deal with plays of finite cost, which do not exist in finitary and bounded parity games.

We begin by relating the winning regions of a parity game with costs and the bounded parity game with costs in the same arena.

Lemma 4.1. Let $\mathcal{G}=(\mathcal{A}, \operatorname{CostParity}(\Omega$, Cst $))$ and let $\mathcal{G}^{\prime}=(\mathcal{A}, \operatorname{BndCostParity}(\Omega$, Cst $))$.

(1) $W_{0}\left(\mathcal{G}^{\prime}\right) \subseteq W_{0}(\mathcal{G})$.

(2) If $W_{0}\left(\mathcal{G}^{\prime}\right)=\emptyset$, then $W_{0}(\mathcal{G})=\emptyset$.

Proof. (11) This follows from the inclusion $\operatorname{Bnd} \operatorname{CostParity}(\Omega$, Cst $) \subseteq \operatorname{CostParity}(\Omega$, Cst).

(2) Due to determinacy, if $W_{0}\left(\mathcal{G}^{\prime}\right)=\emptyset$, then we have $W_{1}\left(\mathcal{G}^{\prime}\right)=V$. Due to Corollary 3.4,

Player 1 has a uniform finite-state strategy $\tau^{\prime}$ that is winning from every vertex $v$ in $\mathcal{G}^{\prime}$. Consider a play consistent with $\tau^{\prime}$ : either, for every $b \in \mathbb{N}$, there is a request that is open for the next $b$ increment-edges (the request could be the same for every $b$ ), or the maximal color seen infinitely often is odd (i.e., there are infinitely many unanswered requests).

To win in the cost-parity game, Player 1 has to keep for every $b$ a different request open for at least $b$ increment-edges (or violate the parity condition). We define a strategy $\tau$ for Player 1 in $\mathcal{G}$ that achieves this by restarting $\tau^{\prime}$ every time a bound $b$ is exceeded. To this end, $\tau$ is guided by a counter $b$ which is initialized with 1 and the strategy $\tau$ behaves like $\tau^{\prime}$ until a request is open for $b$ increment-edges. If this is the case, $b$ is incremented and $\tau$ behaves like $\tau^{\prime}$ does when it starts from the current vertex (forgetting the history of the play constructed so far).

Formally, $\tau$ is implemented by the infinite memory structure $\mathcal{M}=(M$, Init, Upd) where $M=\mathbb{N} \times V^{+}, \operatorname{Init}(v)=(1, v)$, and $\operatorname{Upd}((b, w), v)$ is defined as follows: if $w$ contains a request that is open for more than $b$ increment-edges, then $\operatorname{Upd}((b, w), v)=(b+1, v)$, i.e., the counter is incremented and the play prefix in the second component is reset. On the other 
hand, if $w$ does not contain a request that is open for more than $b$ increment-edges, then $\operatorname{Upd}((b, w), v)=(b, w v)$, i.e., the counter is unchanged and the vertex $v$ is appended to the play prefix. Note that we always have $\operatorname{Upd}^{*}\left(\rho_{0} \cdots \rho_{k}\right)=\left(b, \rho_{k^{\prime}} \cdots \rho_{k}\right)$ for some non-empty suffix $\rho_{k^{\prime}} \cdots \rho_{k}$ of $\rho_{0} \cdots \rho_{k}$. Hence, we can define $\tau\left(\rho_{0} \cdots \rho_{k}\right)=\tau^{\prime}\left(\rho_{k^{\prime}} \cdots \rho_{k}\right)$.

We show that $\tau$ is winning in $\mathcal{G}$ from every vertex, which implies $W_{0}(\mathcal{G})=\emptyset$. Let $\rho$ be a play that is consistent with $\tau$ and distinguish two cases: if the counter in the first component of the memory states reached during $\rho$ is incremented infinitely often, then $\rho$ contains for every $b \in \mathbb{N}$ a request that is open for at least $b$ increment-edges, so $\rho \notin \operatorname{CostParity}(\Omega$, Cst). On the other hand, if the counter is incremented only finitely often (say to value $b$ ), then there is a suffix $\rho^{\prime}$ of $\rho$ that is consistent with the strategy $\tau^{\prime}$. Since the counter is not incremented during $\rho^{\prime}$, every request in $\rho^{\prime}$ is either answered with cost at most $b$ or not answered, but only followed by at most $b$ increment-edges. Hence $\tau^{\prime}$ ensures that the maximal color seen infinitely often in $\rho^{\prime}$ is odd, i.e., $\rho^{\prime} \notin \operatorname{Parity}(\Omega)$, so $\rho \notin \operatorname{Parity}(\Omega)$, and a fortiori $\rho \notin \operatorname{CostParity}(\Omega$, Cst $)$.

We have seen in Example 2.4 that Player 1 needs infinite memory to win cost-parity games. Indeed, the winning strategy for Player 1 described in the example proceeds as the strategy $\tau$ described above. It requests color 1 at vertex $a$, uses the loop at vertex $b$ to keep the request unanswered for several steps and then forgets about this request. At this point, a new request has to be raised by moving from $b$ back to $a$, thereby answering the old request at vertex $c$. This request is then kept unanswered for more increment-edges than the previous one, and this goes on ad infinitum.

To conclude this subsection, we show how Lemma 4.1 can be used to solve parity games with costs. Let $\mathcal{G}=(\mathcal{A}, \operatorname{CostParity}(\Omega, \mathrm{Cst}))$. The following algorithm proceeds by iteratively removing parts of $\mathcal{A}$ that are included in the winning region of Player 0 in $\mathcal{G}$ : we have just proven that the winning region of Player 0 in the bounded parity game with costs in $\mathcal{A}$ is a subset of her winning region in the parity game with costs in the same arena. Thus we can remove it and its attractor. This is repeated until Player 0's winning region in the bounded parity game with costs is empty. In this case, her winning region in the parity game with costs is empty as well, again due to Lemma 4.1. This idea is implemented in the following algorithm.

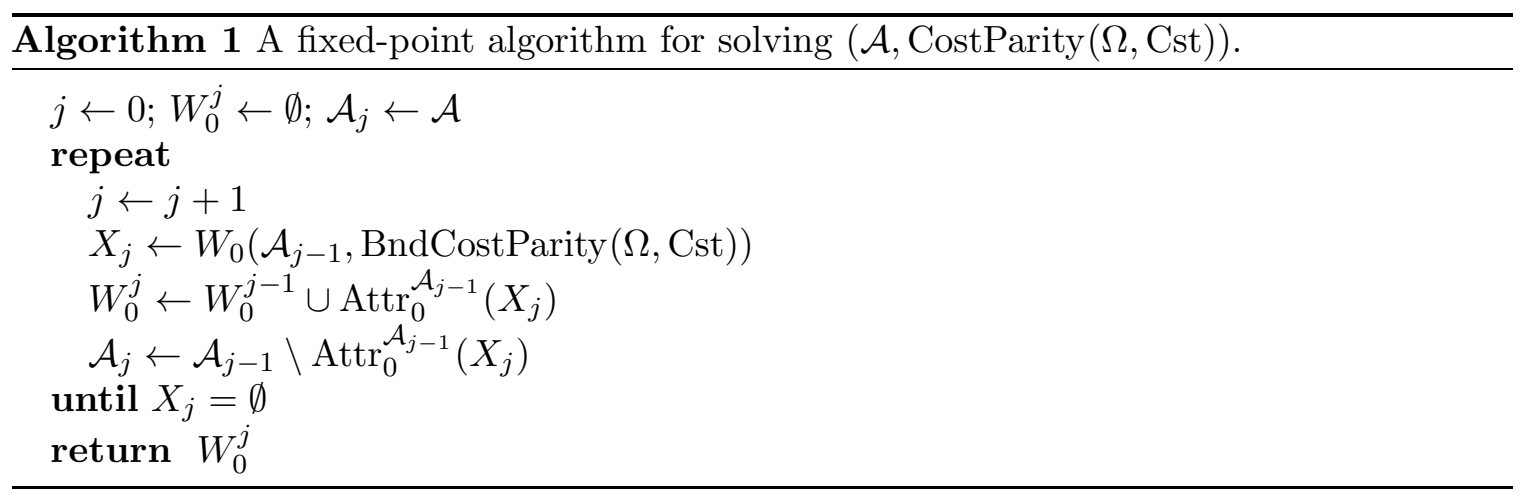

Example 4.2. Running on the parity game with costs of Figure 1, Algorithm 1 computes $X_{1}=\{g\}$ and $W_{0}^{1}=\{f, g\}, X_{2}=\{e\}$ and $W_{0}^{2}=\{d, e, f, g\}$, and $X_{3}=\emptyset$. Thus, it returns $W_{0}^{2}$, which is the winning region of Player 0 in the game.

Next, we show the algorithm to be correct and bound its number of iterations. 
Lemma 4.3. Let $\mathcal{G}$ be a parity game with costs with $n$ vertices. Algorithm 1 returns the winning region $W_{0}(\mathcal{G})$ after at most $n+1$ iterations.

Proof. Let $\mathcal{G}=\left(\left(V, V_{0}, V_{1}, E\right)\right.$, CostParity $(\Omega$, Cst $\left.)\right)$ and let $t$ be the last iteration of Algorithm 1 with $X_{t} \neq \emptyset$, i.e., the algorithm returns $W_{0}^{t}$. We have $t \leq|V|$, since $\emptyset=W_{0}^{0} \subsetneq$ $W_{0}^{1} \subsetneq \cdots \subsetneq W_{0}^{t} \subseteq V$ is a strictly increasing chain. Hence, the algorithm terminates after at most $|V|+1$ iterations.

Next, we show $W_{0}^{t} \subseteq W_{0}(\mathcal{G})$ : to this end, we define a strategy $\sigma$ for Player 0 on $W_{0}^{t}$ as follows: on the sets $X_{j}$ computed by the algorithm, which are winning regions of Player 0 in a bounded parity game with costs, we play using some uniform positional winning strategy for this game, which always exists due to Lemma 3.7. On the attractors $\operatorname{Attr}_{0}^{\mathcal{A}_{j-1}}\left(X_{j}\right)$ we play using some positional attractor strategy. Thus, $\sigma$ is a positional strategy that is defined for every vertex in $W_{0}^{t}$. Next, we show that it is indeed a uniform positional winning strategy from $W_{0}^{t}$.

Every winning region $X_{j}$ is a trap for Player 1 in $\mathcal{A}_{j-1}$. Hence, in the whole arena $\mathcal{A}$, Player 1 can leave $X_{j}$ only to vertices in some $W_{0}^{j^{\prime}}$ with $j^{\prime}<j$. Player 0 on the other hand only moves to vertices in $W_{0}^{j}$. Similarly, if the play is in the attractor of some $X_{j}$, then it reaches $X_{j}$ after at most $|V|$ steps or Player 1 moves to some $W_{0}^{j^{\prime}}$ for some $j^{\prime}<j$. Hence, every play $\rho$ consistent with $\sigma$ has a suffix $\rho^{\prime}$ that visits only vertices from some $X_{j}$ and is consistent with the winning strategy for the corresponding bounded parity game with costs. So, $\rho^{\prime} \in \operatorname{BndCostParity}(\Omega$, Cst), which implies $\rho \in \operatorname{CostParity}(\Omega$, Cst). Thus, $\sigma$ is a winning strategy for Player 0 in $\mathcal{G}$ from $W_{0}^{t}$.

It remains to consider Player 1, i.e., to show that $V \backslash W_{0}^{t} \subseteq W_{1}(\mathcal{G})$. Note that $V \backslash W_{0}^{t}$ is the set of vertices of $\mathcal{A}_{t}$ and that we have $W_{0}\left(\mathcal{A}_{t}, \operatorname{BndCostParity}(\Omega\right.$, Cst $\left.)\right)=\emptyset$. Hence, by Lemma 4.1(2) we conclude $W_{0}\left(\mathcal{A}_{t}, \operatorname{CostParity}(\Omega\right.$, Cst $\left.)\right)=\emptyset$, i.e., Player 1 wins the parity game with costs in the arena $\mathcal{A}_{t}$ from every vertex. Since $V \backslash W_{0}^{t}$ is a trap for Player 0 (it is the complement of an attractor), it follows that Player 1 wins the parity game with costs in the arena $\mathcal{A}$ from every vertex in $V \backslash W_{0}^{t}$.

Corollary 4.4. In parity games with costs, Player 0 has uniform positional winning strategies.

Using Lemma 4.3, Theorem 3.5, and the fact that Algorithm 1 terminates after at most $n+1$ iterations, and therefore has to solve at most $n$ bounded parity games with costs, we obtain the following result where again $n$ is the number of vertices, $m$ is the number of edges, and $d$ is the number of colors in the game.

Theorem 4.5. Given an algorithm that solves parity games in time $T(n, m, d)$, there is an algorithm that solves parity games with costs in time $O(n \cdot T(d n, d m, d+2))$.

Also, we obtain the same computational complexity as for bounded parity games with costs. Here, we rely on the characterization of the winning region $W_{0}(\mathcal{G})$ of a parity game with costs $\mathcal{G}$ as computed by Algorithm 1: the sets $X_{j}$ can be determined in NP (respectively in coNP) due to Remark 3.6 and the attractors can be computed in (deterministic) linear time (note that this was recently improved to $\mathbf{U P} \cap$ coUP [27]).

Remark 4.6. The following problem is in NP $\cap$ coNP: given a parity game with costs $\mathcal{G}$, $i \in\{0,1\}$, and a vertex $v$, is $v \in W_{i}(\mathcal{G})$ ?

In the previous section we have shown that one can recover a polynomial time algorithm for deciding the winning regions of bounded parity games. Hence, using Algorithm 1, we 
obtain the same for finitary parity games as well. Hence, we also recover polynomial time decidability of finitary parity games as a special case of our algorithm. However, this is not surprising since Algorithm 1 is the same one used in [11] to solve finitary parity games via solving bounded parity games.

\section{Positional Winning Strategies for Bounded Parity Games with Costs VIA SCORING FunCtions}

In Lemma 3.7, we have shown how to eliminate the memory introduced in the reduction from PCRR games to parity games, which proved the existence of uniform positional winning strategies for Player 0 in bounded parity games with costs. Using these strategies as building blocks, we also proved the existence of uniform positional winning strategies for Player 0 in parity games with costs. Intuitively, the memory used in the reduction from bounded parity with costs to PCRR keeps track of the largest open request, but Player 0 does not need this information to implement her winning strategy as proved in Lemma 3.7, Instead, she can always play assuming the worst situation that still allows her to win. Thus, we have shown that the memory introduced by this reduction can always be eliminated.

In this section we generalize this construction to memory structures that are not necessarily of the form used in the reduction: we show how to turn an arbitrary uniform finite-state winning strategy for Player 0 in a bounded parity game with costs into a positional one. To this end, we define a quality measure for play prefixes and then show that always playing like in the worst possible situation is a positional winning strategy. This gives an alternative proof of half-positional determinacy of (bounded) parity games with costs and presents a general framework that we believe to be applicable to other winning conditions as well.

We begin by defining a so-called scoring function for bounded parity games with costs that measures the quality of a play prefix (from Player 0's vantage point) by keeping track of the largest unanswered request, the number of increment-edges traversed since it was raised, and how often each odd color was seen since the last increment-edge. This information is gathered in a so-called score-sheet, which is then used to measure the quality of the play prefix. We begin by defining score sheets.

For the remainder of this section, we fix a bounded parity game with costs $\mathcal{G}=$ $(\mathcal{A}$, BndCostParity $(\Omega$, Cst $))$ with arena $\mathcal{A}=\left(V, V_{0}, V_{1}, E\right)$, and an arbitrary uniform finitestate winning strategy $\sigma$ for Player 0 in $\mathcal{G}$ which we want to turn into a uniform positional winning strategy. Let $\Omega(V) \subseteq\{0,1, \ldots, \ell\}$, where we assume $\ell$ to be odd. Furthermore, let $d=\frac{\ell+1}{2}$ be the number of odd colors in $\{0,1, \ldots, \ell\}$. Finally, let $t=|V| \cdot|\sigma|$, where $|\sigma|$ denotes the size of the memory structure implementing $\sigma$.

A proper (score-) sheet is a vector $\left(c, n, s_{\ell}, s_{\ell-2}, \ldots, s_{3}, s_{1}\right)$ where $c$ is an odd color in $\{1,3, \ldots, \ell\}, n \leq t$, and $s_{c^{\prime}} \leq t$ for every $c^{\prime}$. Finally, we use two non-proper sheets denoted by $\perp$ and $T$. The reversed ordering of the score values $s_{\ell}, s_{\ell-2}, \ldots, s_{3}, s_{1}$ in the sheets is due to the max-parity condition, in which larger colors are more important than smaller ones. This is reflected by the fact that we compare sheets in the lexicographical order induced by $<$ on its components and add $\perp$ as minimal and $T$ as maximal element. For example, $(3,3,0,1,1)<(3,3,1,0,3)$ and $\perp<s<\top$ for every sheet $s \neq \perp, \top$. As usual, we write $s \leq s^{\prime}$ if $s=s^{\prime}$ or $s<s^{\prime}$.

\footnotetext{
${ }^{4} \mathrm{~A}$ game is half-positionally determined, if one of the players has a positional winning strategy from every vertex of her winning region.
} 
Next, we show how to update sheets along a play to use them as a quality measure for play prefixes. Let $s=\left(x_{1}, \ldots, x_{d+2}\right)$ be a proper sheet. We say that $s$ is full in coordinate 1 , if $x_{1}=\ell$ (recall that $\ell$ is the largest possible value in the first coordinate), and that $s$ is full in coordinate $k>1$, if $x_{k}=t$ (recall that $t$ is the largest possible value in all but the first coordinate). Let $k$ be a coordinate and let $s=\left(x_{1}, \ldots, x_{d+2}\right)$ be a proper sheet.

- If 1 is the largest coordinate smaller than or equal to $k$ that is not full in $s$, then incrementing $s$ at coordinate $k$ yields the sheet $\left(x_{1}+2,0, \ldots, 0\right)$. If $k>1$, then we say that there is an overflow in coordinates $2, \ldots, k$.

- If $k^{\prime}>1$ is the largest coordinate smaller than or equal to $k$ that is not full in $s$, then incrementing $s$ at coordinate $k$ yields the sheet $\left(x_{1}, \ldots, x_{k^{\prime}-1}, x_{k^{\prime}}+1,0, \ldots, 0\right)$. If $k^{\prime}<k$, then we say that there is an overflow in coordinates $k^{\prime}+1, \ldots, k$.

- If there is no coordinate $k^{\prime}$ smaller than or equal to $k$ that is not full in $s$, then incrementing $s$ at coordinate $k$ yields the sheet $T$ and we say that there is an overflow in coordinates $1, \ldots, k$.

Example 5.1. Assume we have $\ell=5$ and $t=3$ and consider $s=(3,3,0,1,3)$. Then, $s$ is full in coordinate 2 and 5 , but not in coordinates 1,3 , and 4 . Incrementing $s$ at coordinate 1 or 2 yields the sheet $(5,0,0,0,0)$ (note that there is an overflow of coordinate 2 in the second case), incrementing at 3 yields $(3,3,1,0,0)$ while incrementing at 4 or 5 yields $(3,3,0,2,0)$ (and there is an overflow of coordinate 5 in the second case).

Next, we show that the increment-operation and a reset-operation are compatible with the ordering. Recall that we compare sheets lexicographically.

Remark 5.2. Let $x=\left(x_{1}, \ldots, x_{d+2}\right) \leq y=\left(y_{1}, \ldots, y_{d+2}\right)$ be two sheets and let $k$ be a coordinate.

(1) Let $x^{\prime}$ (respectively $y^{\prime}$ ) be obtained by incrementing $x$ (respectively $y$ ) at coordinate $k$. Then, $x^{\prime} \leq y^{\prime}$.

(2) Let $x^{\prime \prime}=\left(x_{1}, \ldots, x_{k}, 0, \ldots, 0\right)$ and $y^{\prime \prime}=\left(y_{1}, \ldots, y_{k}, 0, \ldots, 0\right)$. Then, $x^{\prime \prime} \leq y^{\prime \prime}$.

Now, we want to assign a sheet to every play prefix. To this end, we define the initial sheet $\operatorname{Sh}(v)$ of a vertex $v$ by

$$
\operatorname{Sh}(v)= \begin{cases}\perp & \text { if } \Omega(v) \text { is even, } \\ (\Omega(v), 0,0, \ldots, 0) & \text { if } \Omega(v) \text { is odd. }\end{cases}
$$

Now, let $\operatorname{Sh}(w v)$ for $w \in V^{*}$ and $v \in V$ be already defined and let $\left(v, v^{\prime}\right)$ be an edge. If $\operatorname{Sh}(w v)=\top$, then $\operatorname{Sh}\left(w v v^{\prime}\right)=\top$, and if $\operatorname{Sh}(w v)=\perp$, then $\operatorname{Sh}\left(w v v^{\prime}\right)=\operatorname{Sh}\left(v^{\prime}\right)$. Now, assume we have $\operatorname{Sh}(w v)=\left(c, n, s_{\ell}, \ldots, s_{1}\right)$, i.e., $c$ is the largest open request in $w v$. We have to distinguish several cases.

- If $\Omega\left(v^{\prime}\right)>c$, then $\operatorname{Sh}\left(w v v^{\prime}\right)=\operatorname{Sh}\left(v^{\prime}\right)$, i.e., if $\Omega\left(v^{\prime}\right)$ is even larger than $c$ and odd, then the first component is updated to $\Omega\left(v^{\prime}\right)$ and all others are reset to zero. If $\Omega\left(v^{\prime}\right)$ is even and larger than $c$, then all requests are answered and the sheet is reset to $\perp$.

- If $\Omega\left(v^{\prime}\right) \leq c$ and $\operatorname{Cst}\left(v, v^{\prime}\right)=i$ (i.e., the largest open request is still $c$ but an incrementedge is traversed), then $\operatorname{Sh}\left(w v v^{\prime}\right)$ is obtained from $\operatorname{Sh}(w v)$ by incrementing the second coordinate (the one associated with costs).

- if $\Omega\left(v^{\prime}\right) \leq c, \operatorname{Cst}\left(v, v^{\prime}\right)=\varepsilon$ and $\Omega\left(v^{\prime}\right)$ even, then the scores for the colors that are answered by $\Omega\left(v^{\prime}\right)$ are reset to zero, i.e., $\operatorname{Sh}\left(w v v^{\prime}\right)=\left(c, n, s_{\ell}, \ldots, s_{\Omega\left(v^{\prime}\right)+1}, 0, \ldots, 0\right)$,

- if $\Omega\left(v^{\prime}\right) \leq c, \operatorname{Cst}\left(v, v^{\prime}\right)=\varepsilon$ and $\Omega\left(v^{\prime}\right)$ odd, then $\operatorname{Sh}\left(w v v^{\prime}\right)$ is obtained from $\operatorname{Sh}(w v)$ by incrementing the coordinate storing the score for $\Omega\left(v^{\prime}\right)$. 
Note that the increments in the second and fourth case of the definition might trigger overflows in case the respective coordinates are full in $\mathrm{Sh}(w v)$.

Let $\operatorname{Sh}(w)=\left(c, n, s_{\ell}, \ldots, s_{1}\right)$. To simplify our notation in the following proofs, we define $\operatorname{Req}(w)=c, \operatorname{ReqCst}(w)=n$, and $\operatorname{Sc}_{c^{\prime}}(w)=s_{c^{\prime}}$. If $\operatorname{Sh}(w)=\perp$ or $\operatorname{Sh}(w)=\top$, then we leave these functions undefined. Furthermore, let Lst $(w)$ denote the last vertex of a non-empty finite play $w$.

In the following, we show three properties of the scoring function that are used to prove our main result. We begin by showing that it is a congruence.

Lemma 5.3. If $\operatorname{Lst}(x)=\operatorname{Lst}(y)$ and $\operatorname{Sh}(x) \leq \operatorname{Sh}(y)$, then $\operatorname{Sh}(x v) \leq \operatorname{Sh}(y v)$ for every $v \in V$.

Before we begin the proof we state the following useful facts.

Remark 5.4. Let $w \in V^{*}$ and $v \in V$.

(1) If $\operatorname{Req}(w) \neq \operatorname{Req}(w v)$, then $\operatorname{Sh}(w v)=\operatorname{Sh}(v)$.

(2) If $\Omega(v)$ is odd, then $\operatorname{Sh}(w v) \geq \operatorname{Sh}(v)$.

Now, we are ready to prove Lemma 5.3 .

Proof. If $\operatorname{Sh}(x)=\operatorname{Sh}(y)$, then $\operatorname{Sh}(x v)=\operatorname{Sh}(y v)$, since the sheets of $x v$ and $y v$ only depend on the sheets of $x$ and $y$ (which are equal) and the last edges of $x v$ and $y v$ (which are also equal).

So, consider the case $\operatorname{Sh}(x)<\operatorname{Sh}(y)$. First, assume we have $\operatorname{Sh}(x)=\perp$, which implies $\operatorname{Sh}(x v)=\operatorname{Sh}(v)$. If $\Omega(v)$ is even, then $\operatorname{Sh}(v)=\perp$ and we are done, since $\perp$ is the minimal element. Otherwise, applying Remark 5.4(2) to yv yields the desired result. As a last special case assume we have $\operatorname{Sh}(y)=\top$, which implies $\operatorname{Sh}(y v)=\top$. As $\top$ is the maximal element, we have $\operatorname{Sh}(x v) \leq \operatorname{Sh}(y v)$.

We are left with the case $\perp<\mathrm{Sh}(x)<\mathrm{Sh}(y)<\top$ and have to consider two subcases:

(1) First, assume we have $\operatorname{Req}(x)=\operatorname{Req}(y)$. We consider several subcases.

(a) If $\Omega(v)>\operatorname{Req}(x)=\operatorname{Req}(y)$, then $\operatorname{Sh}(x v)=\operatorname{Sh}(y v)=\operatorname{Sh}(v)$.

(b) If $\Omega(v) \leq \operatorname{Req}(x)=\operatorname{Req}(y)$ and $\operatorname{Cst}(\operatorname{Lst}(x), v)=i$, then both $\operatorname{Sh}(x v)$ and $\operatorname{Sh}(y v)$ are obtained by incrementing $\operatorname{Sh}(x)$ and $\operatorname{Sh}(y)$ respectively at the second coordinate. Hence, Remark 5.2(1) yields the desired result.

(c) If $\Omega(v) \leq \operatorname{Req}(x)=\operatorname{Req}(y), \operatorname{Cst}(\operatorname{Lst}(x), v)=\varepsilon$, and $\Omega(v)$ is even, then both $\operatorname{Sh}(x v)$ and $\operatorname{Sh}(y v)$ are obtained by reseting the scores for every $c^{\prime}$ smaller than $\Omega(v)$. Hence, Remark 5.2(2) yields the desired result.

(d) If $\Omega(v) \leq \operatorname{Req}(x)=\operatorname{Req}(y), \operatorname{Cst}(\operatorname{Lst}(x), v)=\varepsilon$, and $\Omega(v)$ is odd, then both $\operatorname{Sh}(x v)$ and $\operatorname{Sh}(y v)$ are obtained by incrementing $\operatorname{Sh}(x)$ and $\operatorname{Sh}(y)$ respectively at the coordinate storing the score for $\Omega(v)$. Hence, Remark 5.2(1) yields the desired result.

(2) Now, assume we have $\operatorname{Req}(x)<\operatorname{Req}(y)$. Note that $\operatorname{Sh}(x v)=\top$ is impossible in this case, since the first coordinate of $x$ is not full, as it is strictly smaller than $\operatorname{Req}(y)$. We again have to consider several subcases:

(a) If $\Omega(v)>\operatorname{Req}(y)>\operatorname{Req}(x)$, then $\operatorname{Sh}(x v)=\operatorname{Sh}(y v)=\operatorname{Sh}(v)$.

(b) If $\Omega(v)=\operatorname{Req}(y)>\operatorname{Req}(x)$, then we have $\operatorname{Req}(x v)>\operatorname{Req}(x)$ and an application of Remark 5.4(1) and 5.4(2) (to $y v$ ) yields the desired result.

(c) Finally, assume we have $\Omega(v)<\operatorname{Req}(y)$. We again have to consider three subcases.

(i) If we have $\operatorname{Sh}(y v)=\top$, then we are done.

(ii) Assume we have $\operatorname{Req}(y v)>\operatorname{Req}(y)$. Then the following inequalities hold: $\operatorname{Req}(x v) \leq \operatorname{Req}(x)+2 \leq \operatorname{Req}(y)<\operatorname{Req}(y v)$, where the first one is due 
to the fact that the first component of $\operatorname{Sh}(x v)$ can only increase due to an increment, and the second one due to $\operatorname{Req}(x)<\operatorname{Req}(y)$. Hence, we have $\operatorname{Sh}(x v)<\operatorname{Sh}(y v)$ in this case.

(iii) Finally, consider the case where $\operatorname{Req}(y v)=\operatorname{Req}(y)$. If $\operatorname{Req}(x v)<\operatorname{Req}(y v)$, then we are done. So, assume we have $\operatorname{Req}(x v)=\operatorname{Req}(y v)$. Then, the first component of $\operatorname{Sh}(x)$ is increased to obtain $\operatorname{Sh}(x v)$, which implies that all other components of $\operatorname{Sh}(x v)$ are equal to zero. Hence, we have $\operatorname{Sh}(x v) \leq \operatorname{Sh}(y v)$.

We continue by showing that the sheets of a play $\rho$ being bounded is a sufficient condition for $\rho$ satisfying the bounded cost-parity condition.

Lemma 5.5. If the sheets of all prefixes of a play $\rho$ are strictly smaller than $T$, then $\rho \in \operatorname{Bnd} \operatorname{CostParity}(\Omega$, Cst).

Proof. We prove the converse, i.e., if $\rho \notin \operatorname{BndCostParity}(\Omega$, Cst $)$, then there is a prefix of $\rho$ whose sheet is $T$. First, assume that for every $b$ there is a request (say of color $c$ ) that is open for at least $b$ increment-edges. Then, the second component of the sheets is incremented every time an increment-edge is traversed before the request is answered. Also, the first component is increased every time the second component overflows or every time a larger odd color is visited. Note that it is not reset in this interval, as the request of $c$ is not answered. Hence, if we pick $b$ large enough, the first component overflows as well. This yields the sheet $T$.

Now assume the maximal color seen infinitely often, call it $c$, is odd. We may assume that $\rho$ has only finitely many increment-edges, as we are in the first case otherwise. Pick a position of $\rho$ such that the maximal color appearing after this position is $c$ and such that no increment-edge is traversed after this position. After this position, the coordinate storing the score for $c$ is incremented again and again. Furthermore, this coordinate (and all to the left of this one) are only reset in case of an overflow, which means that there is a coordinate to the left that is incremented. Thus, every coordinate to the left of the one storing the score for $c$ is incremented again and again, too. Hence, the first component overflows at some point, which yields the sheet $T$.

Recall that the entries in all but the first component of a (proper) sheet are bounded by $t=|V| \cdot|\sigma|$, where $\sigma$ is a uniform finite-state winning strategy for Player 0 in $\mathcal{G}=$ $(\mathcal{A}, \operatorname{BndCostParity}(\Omega$, Cst $))$. Next, we show that this strategy keeps the sheets smaller than $T$.

Lemma 5.6. Let $\rho$ be starting in $W_{0}(\mathcal{G})$ and consistent with $\sigma$. Then, the sheets of all prefixes of $\rho$ are strictly smaller than $\top$.

Proof. First, we show that every request in $\rho$ is answered with cost less than or equal to $t$ or followed by at most $t$ increment-edges. Towards a contradiction, assume there is a request that is followed by $t+1$ increment-edges, but no answer before the last of these increment-edges. Then, there are two positions in this interval that have the same vertex, the memory structure implementing $\sigma$ assumes the same state after both positions, and there is at least one increment-edge between these positions. Hence, there is also a play consistent with $\sigma$ and starting in $W_{0}(\mathcal{G})$ that contains an unanswered request with cost $\infty$. However, this contradicts the fact that $\sigma$ is a winning strategy.

Similarly, one can show that $\rho$ has no infix that contains $t+1$ vertices of some odd color $c$, but no vertex of a larger color. Using the second property, a simple induction over 
the number of odd colors (starting with 1 ) shows that no coordinate storing a score $s_{c}$ overflows during $\rho$. Using this and the first property shows that the second coordinate does not overflow either. Finally, if the second component does not overflow, then the first component does not overflow either, since it stores the largest unanswered request in this case. Hence, the sheets of $\rho$ are strictly smaller than $T$.

Now we are able to prove our main technical result of this section: using the scoresheets we can turn an arbitrary uniform finite-state winning strategy into a positional one. For every $v \in V$, let $P_{v}$ denote the set of play prefixes that begin in $W_{0}(\mathcal{G})$, are consistent with $\sigma$, and end in $v$. Due to Lemma 5.6, the sheets of the prefixes in $P_{v}$ are strictly smaller than $\top$. Hence, for every nonempty $P_{v}$ there exists a play prefix $\max _{v} \in P_{v}$ such that $\operatorname{Sh}(w) \leq \operatorname{Sh}\left(\max _{v}\right)<\top$ for every $w \in P_{v}$. We define a positional strategy $\sigma^{\prime}$ by $\sigma^{\prime}(w v)=\sigma\left(\max _{v}\right)$.

Lemma 5.7. The strategy $\sigma^{\prime}$ is a uniform positional winning strategy for Player 0 in $\mathcal{G}$.

Proof. An inductive application of Lemma 5.3 shows that we have $\operatorname{Sh}\left(\rho_{0} \cdots \rho_{n}\right) \leq \operatorname{Sh}\left(\max _{\rho_{n}}\right)$ for every $n$ and every play $\rho$ that is consistent with $\sigma^{\prime}$. Hence, the sheets of $\rho$ are strictly smaller than $\top$, which implies $\rho \in \operatorname{Bnd} \operatorname{CostParity}(\Omega)$ due to Lemma 5.5.

In the preliminary version of this work [20], we presented a similar construction, the main difference being that we did not use overflows there, but updated a sheet to $T$ if a full coordinate is incremented. This construction can also shown to be correct, but the proof of the analogue of Lemma 5.7 in [20] (called Lemma 15 there) has a gap (the claim in its last line is incorrect). This gap can be closed using pumping arguments which rely on properties of the bounded cost-parity condition. Since one of our aims in this section is to give a general framework that works for other winning conditions as well, we refrained from presenting the fix and instead changed the definition of the sheets (adding overflows) to achieve this goal. Indeed, our construction only relies on the following properties:

(1) The score-sheets constitute a finite total order.

(2) The score-sheet function is a congruence w.r.t. this order.

(3) If the score-sheets of a play are strictly smaller than the maximal element, then it is winning for Player 0.

(4) A finite-state winning strategy allows only plays whose score-sheets are strictly smaller than the maximal element.

It follows that for every winning condition for which one can define a scoring function meeting these conditions, one can turn a finite-state winning strategy into a positional one. For example, one could extend the sheets presented above by a new first coordinate that counts how often the second coordinate (the largest open request) overflows, which corresponds to requests that are open for many increment-edges. Since a finite-state winning strategy for a parity game with costs bounds the number of such requests, our framework is applicable to parity games with costs as well.

On the other hand, our framework cannot applicable to (bounded) Streett games with costs since they are not positionally determined. This impossibility manifests itself in the fact that one cannot totally order the costs of the different requests of a Streett condition while satisfying the other three properties listed above. This is in contrast to (bounded) parity games with costs where larger requests are more important than smaller ones, since answering the larger ones also answers smaller ones. This is reflected in the lexicographic ordering of the sheets. Interestingly, our result above relies on having just one cost function 
that is used for every request. If we allow different cost functions for different colors, then both players need memory to implement their winning strategies (cf. Section 7), i.e., our framework cannot be applicable.

Finally, by relaxing the first requirement (the score-sheets being totally ordered) to allow the sheets being partially ordered, then one obtains a finite-state winning strategy whose size is at most the size of the largest anti-chain in the partial order of the sheets (see [30] for an application of this idea).

\section{Streett Games with Costs}

In this section, we present an algorithm to solve Streett and bounded Streett games with costs following the same ideas as in the section about (bounded) parity games with costs, and prove EXPTIME-completeness of the corresponding decision problems. From our algorithm, we also obtain upper bounds on the memory requirements of both players, which are complemented by lower bounds.

The main result of this section is the following theorem. Here, $n$ is the number of vertices, $m$ is the number of edges, and $d$ is the number of Streett pairs in the game.

Theorem 6.1. Given an algorithm that solves Streett games in time $T(n, m, d)$, there is

(1) an algorithm that solves bounded Streett games with costs in time $O\left(T\left(2^{d} n, 2^{d} m, 2 d\right)\right)$.

(2) an algorithm that solves Streett games with costs in time $O\left(n \cdot T\left(2^{d} n, 2^{d} m, 2 d\right)\right)$.

Fix an arena $\mathcal{A}=\left(V, V_{0}, V_{1}, E\right)$, a collection $\Gamma=\left(Q_{c}, P_{c}\right)_{c \in[d]}$ of Streett pairs, and a collection $\overline{\mathrm{Cst}}=\left(\mathrm{Cst}_{c}\right)_{c \in[d]}$ of cost functions, both compatible with $\mathcal{A}$. We begin by considering bounded games and again assume for every $c \in[d]$ that no vertex of $\mathcal{A}$ has both an incoming increment-edge (w.r.t. Cst $_{c}$ ) and an incoming $\varepsilon$-edge (again, w.r.t. Cst ${ }_{c}$ ). Having different types of incoming edges with respect to different cost functions is allowed. This property can again be established by subdividing edges. Assuming this, let $I_{c}$ denote the vertices with incoming increment-edges w.r.t. $\operatorname{Cst}_{c}$. Then, $\operatorname{coBüchi}\left(I_{c}\right)=\left\{\rho \mid \operatorname{Cst}_{c}(\rho)<\right.$ $\infty\}$ is the set of plays with finitely many increment-edges w.r.t. Cst $_{c}$. Let $I=\left(I_{c}\right)_{c \in[d]}$. Furthermore, we define $\operatorname{RR}\left(Q_{c}, P_{c}\right)$ to be the set of plays in which every request of pair $c$ is eventually answered. Finally, we define

$$
\operatorname{SCRR}(\Gamma, I)=\bigcap_{c \in[d]}\left[\left(\operatorname{Streett}\left(Q_{c}, P_{c}\right) \cap \operatorname{coBüchi}\left(I_{c}\right)\right) \cup \operatorname{RR}\left(Q_{c}, P_{c}\right)\right],
$$

which is $\omega$-regular as a boolean combination of $\omega$-regular languages. This condition is a relaxation of the bounded cost-Streett condition, as we have $\operatorname{SCRR}(\Gamma) \supseteq \operatorname{BndCostStreett}(\Gamma)$.

Lemma 6.2. Let $\mathcal{G}=(\mathcal{A}, \operatorname{BndCostStreett}(\Gamma, \overline{\mathrm{Cst}}))$ and $\mathcal{G}^{\prime}=(\mathcal{A}, \operatorname{SCRR}(\Gamma, I))$, where $I$ is defined as above. A winning strategy for Player $i$ in $\mathcal{G}^{\prime}$ from a set $W$ is also a winning strategy for Player $i$ in $\mathcal{G}$ from $W$. Especially, $W_{i}(\mathcal{G})=W_{i}\left(\mathcal{G}^{\prime}\right)$ for $i \in\{0,1\}$.

The proof of this lemma is similar to the one for Lemma 3.1 and relies on finite-state determinacy of $\omega$-regular games.

Next, we show how to reduce $(\mathcal{A}, \operatorname{SCRR}(\Gamma, I))$ to a classical Streett game: first, we add a memory structure $\mathcal{M}$ of size $2^{d}$ that keeps track of the open requests during a play (cp. [22]) and let $F_{c}$ denote the vertices in which request $c$ is not open. Then, we have 
$(\mathcal{A}, \operatorname{SCRR}(\Gamma, I)) \leq_{\mathcal{M}}(\mathcal{A} \times \mathcal{M}, L)$ with

$$
L=\bigcap_{c \in[d]}\left[\left(\operatorname{Streett}\left(Q_{c}, P_{c}\right) \cap \operatorname{coBüchi}\left(I_{c}\right)\right) \cup \operatorname{Büchi}\left(F_{c}\right)\right],
$$

i.e., we have reduced the request-response conditions $\mathrm{RR}\left(Q_{c}, P_{c}\right)$ to Büchi conditions. Finally, we have

$$
\begin{aligned}
L & =\bigcap_{c \in[d]}\left[\left(\operatorname{Streett}\left(Q_{c}, P_{c}\right) \cap \operatorname{coBüchi}\left(I_{c}\right)\right) \cup \operatorname{Büchi}\left(F_{c}\right)\right] \\
& =\bigcap_{c \in[d]}\left[\left(\operatorname{Streett}\left(Q_{c}, P_{c}\right) \cap \operatorname{Streett}\left(I_{c}, \emptyset\right)\right) \cup \operatorname{Büchi}\left(F_{c}\right)\right] \\
& =\bigcap_{c \in[d]}\left[\left(\operatorname{Streett}\left(Q_{c}, P_{c}\right) \cup \operatorname{Büchi}\left(F_{c}\right)\right) \cap\left(\operatorname{Streett}\left(I_{c}, \emptyset\right) \cup \operatorname{Büchi}\left(F_{c}\right)\right)\right] \\
& =\bigcap_{c \in[d]}\left[\operatorname{Streett}\left(Q_{c}, P_{c} \cup F_{c}\right) \cap \operatorname{Streett}\left(I_{c}, F_{c}\right)\right],
\end{aligned}
$$

which is a Streett condition. Thus, we have reduced $(\mathcal{A}, \operatorname{SCRR}(\Gamma, I))$ to a Streett game in an arena that is exponential in $d$ with $2 d$ Streett pairs. This proves the first claim of Theorem 6.1. Furthermore, we obtain the following upper bound on the size of finite-state winning strategies. Here, we use the fact that Player 0 has finite-state winning strategies of size $d$ ! in Streett games with $d$ pairs (which is tight), while Player 1 has positional winning strategies [16]. Note that the lower bound of $d$ ! for Player 0 is also a lower bound for her in (bounded) Streett games with costs, since classical Streett games are a special case of both.

\section{Remark 6.3.}

(1) In bounded Streett games with costs, Player 0 has uniform finite-state winning strategies of size $2^{d}((2 d)$ !), where $d$ is the number of Streett pairs.

(2) In bounded Streett games with costs, Player 1 has uniform finite-state winning strategies of size $2^{d}$, where $d$ is the number of Streett pairs.

It remains to show a lower bound on the memory requirements for Player 1.

Lemma 6.4. For every $d \geq 1$, there is a bounded Streett game with costs $\mathcal{G}_{d}$ with a designated vertex $v$ such that

- the arena of $\mathcal{G}_{d}$ is of linear size in $d$ and $\mathcal{G}_{d}$ has $2 d$ Streett pairs,

- Player 1 has a uniform finite-state winning strategy for $\mathcal{G}_{d}$ from $v$, which is implemented with $2^{d}$ memory states, but

- Player 1 has no winning strategy from $v$ that is implemented with less than $2^{d}$ memory states.

Proof. The arena $\mathcal{A}_{d}$ of $\mathcal{G}_{d}$ is depicted in Figure 4 where we do not indicate the costs, since every edge is an increment-edge (for every cost function). The winning condition is given as $\Gamma_{d}=\left(Q_{c}, P_{c}\right)_{c \in[2 d]}$ where $Q_{c}=\left\{q_{c}\right\}$ and $P_{c}=\left\{s_{c^{\prime}} \mid c^{\prime} \neq c\right\}$. The designated vertex $v$ we consider is $v_{0}$. Note that every play ends up in one of the sink vertices $s_{c}$.

First, we show that Player 1 has a winning strategy from $v_{0}$ that is implemented with $2^{d}$ memory states. Assume the play is currently ending in vertex $v_{c}^{\prime}$. This play passed through $v_{c}$ where Player 0 either moved to $q_{2 c}$ or to $q_{2 c+1}$. In the first case, Player 1 moves to $p_{2 c}$, in

\footnotetext{
${ }^{5} \operatorname{Büchi}\left(F_{c}\right)$ is the set of plays visiting $F_{c}$ infinitely often.
} 


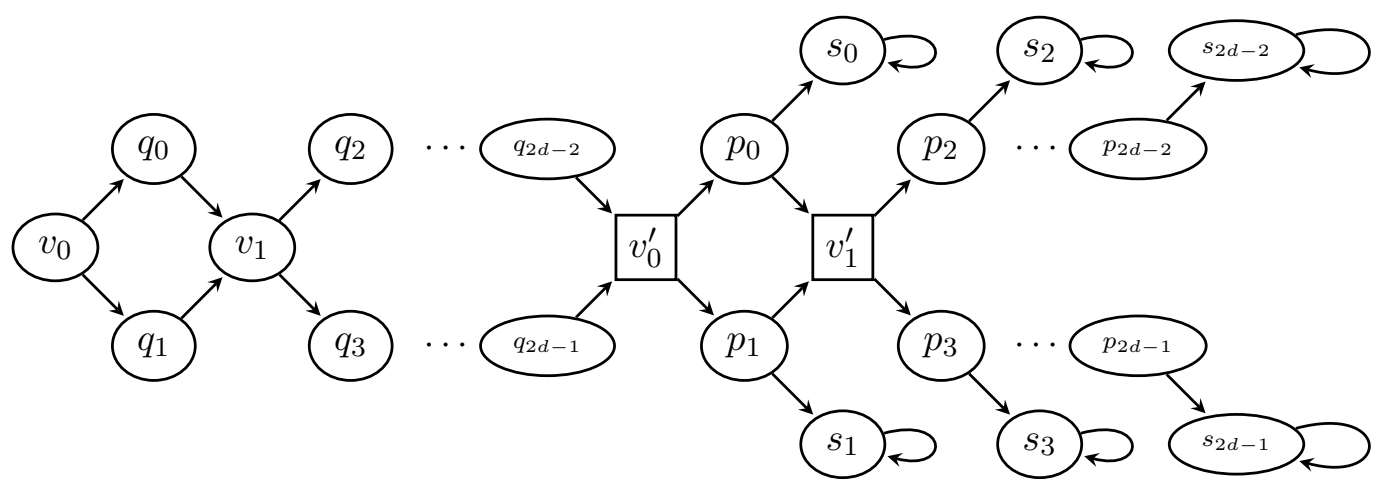

Figure 4: The arena $\mathcal{A}_{d}$ for the bounded Streett game with costs $\mathcal{G}_{d}$ (every edge is an increment-edge).

the second case to $p_{2 c+1}$. Now, consider a play that is consistent with this strategy. It will eventually end up in one of the sink vertices $s_{c}$ after visiting the vertex $p_{c}$, which implies that the vertex $q_{c}$ is visited during the play, too. As $q_{c} \in Q_{c}$, a request of condition $c$ is open, which is never answered, since only sink vertices are in $P_{c}$, but the sink $s_{c}$ is not in $P_{c}$. As every edge is an increment-edge w.r.t. Cst $_{c}$, the play contains an unanswered request of cost $\infty$, i.e., it is winning for Player 1 . Note that this strategy can be implemented by memorizing the $d$ binary choices Player 0 makes at the vertices $v_{j}$, which can be done using $2^{d}$ memory states.

Now, consider a finite-state strategy $\tau$ for Player 1 implemented with less than $2^{d}$ memory states. Then, there are two different play prefixes $w, w^{\prime}$ leading from $v_{0}$ to $v_{0}^{\prime}$ such that the memory structure reaches the same memory state after processing these two prefixes. Since these prefixes differ, there is a $c$ such that $w$ visits $q_{2 c}$ and $w^{\prime}$ visits $q_{2 c+1}$. Now, consider the prolongations of these prefixes where Player 1 plays according to $\tau$ and Player 0 does not move to the sink vertices in order to reach vertex $v_{c}^{\prime}$. Since the memory structure reaches the same memory state after processing $w$ and $w^{\prime}$, it behaves the same after processing these prolongations. Hence, $\tau$ makes the same move after both prolongations, say it moves to $p_{2 c}$ (the case $p_{2 c+1}$ is analogous). Now consider the prolongation of $w^{\prime}$ : Player 1 moves to $p_{2 c}$ and then Player 0 can move to the sink vertex $s_{2 c}$, where the requests of every condition but $2 c$ are answered to. However, a request of condition $2 c$ is not open during this play, since $w^{\prime}$ visited $q_{2 c+1}$ and therefore did not visit $q_{2 c}$. Hence, every request is answered and no new ones are raised in the sink. Hence, the play is winning for Player 0 . Thus, the strategy $\tau$ cannot be winning for Player 1 .

Note that the game $\mathcal{G}_{d}$ presented above is even a bounded Streett game [11.

Now, we consider Streett games with costs: we again show that solving the bounded variant suffices to solve such games.

Lemma 6.5. Let $\mathcal{G}=(\mathcal{A}, \operatorname{CostStreett}(\Gamma, \overline{\mathrm{Cst}}))$ and let $\mathcal{G}^{\prime}=(\mathcal{A}, \operatorname{Bnd} \operatorname{Cost} \operatorname{Streett}(\Gamma, \overline{\mathrm{Cst}}))$.

(1) $W_{0}\left(\mathcal{G}^{\prime}\right) \subseteq W_{0}(\mathcal{G})$.

(2) If $W_{0}\left(\mathcal{G}^{\prime}\right)=\emptyset$, then $W_{0}(\mathcal{G})=\emptyset$. 
The proof is exactly the same as the one for Lemma 4.1. Also, Algorithm 1 (where $X_{j}$ is now Player 0's winning region in the bounded Streett game with costs) works for this pair of winning conditions as well. This proves the second claim of Theorem 6.1,

Furthermore, using the same construction as presented in the proof of Lemma 4.3, one can built a winning strategy for a Streett game with costs out of the winning strategies for the bounded Streett games with costs solved by (the modified) Algorithm 1. By reusing memory states in the different sets $X_{j}$ computed by the algorithm, we obtain the following upper bound for Player 0 . Note that we can reuse memory states, since no information needs to be transferred between the regions $X_{j}$ : once a set $X_{j}$ is entered, the strategy forgets about the history of the play.

Remark 6.6. In Streett games with costs, Player 0 has uniform finite-state winning strategies of size $2^{d}((2 d) !)$, where $d$ is the number of Streett pairs.

Again, the lower bound of $d$ ! for Player 0 in classical Streett games is also a lower bound for her in Streett games with costs. Player 1 on the other hand needs infinite memory in Streett games with costs, as witnessed by the game in Example 2.4, which can be easily transformed into a Streett game with costs.

Using the algorithm presented in [31, which solves a Streett game in time $O\left(m n^{d} d d !\right)$, one can solve (bounded) Streett games with costs in exponential time, although the Streett games that need to be solved are of exponential size (but only in $d$ ). Here it is crucial that the number of Streett pairs only grows linearly. Together with the EXPTIME-hardness of solving bounded and finitary Streett games, which are a special case of (bounded) Streett games with costs, we obtain the following result.

Theorem 6.7. The following problem is EXPTIME-complete: Given a (bounded) Streett game with costs $\mathcal{G}, i \in\{0,1\}$, and a vertex $v$, is $v \in W_{i}(\mathcal{G})$ ?

\section{Conclusion}

We introduced infinite games with cost conditions, generalizing both classical conditions and finitary conditions. For parity games with costs, we proved half-positional determinacy and that solving these games is not harder than solving parity games. The decision problem is in $\mathbf{N P} \cap \mathbf{c o N P}$ (this was recently improved to $\mathbf{U P} \cap \mathbf{c o U P}[27]$ ).

For Streett games with costs, we showed that Player 0 has finite-state winning strategies and that solving these games is not harder than solving finitary Streett games and can be done by solving linearly many (classical) Streett games of exponential size (in the number of Streett pairs). Table 1 sums up all our results on games with costs and compares them to the results for the classical and finitary variants. Here, $d$ denotes the number of odd colors in the game and "exponential" is always meant to be "exponential in the number of Streett-pairs". The memory bounds for the different types of parity games are tight, while there are gaps between the exponential lower and the exponential upper bounds for the different types of Streett games with costs.

Let us discuss two variations of the games presented here. In a parity game with costs, the requests and responses are hierarchical and there is a single cost function that is used for every request. On the other hand, in Streett games with costs, the requests and responses

\footnotetext{
${ }^{6}$ Shown in unpublished work by Chatterjee, Henzinger, and Horn, obtained by slightly modifying the proof of EXPTIME-hardness of solving request-response games [12].
} 


\begin{tabular}{llll}
\hline winning condition & computational complexity & memory Pl. 0 & memory Pl. 1 \\
\hline parity & UP $\cap$ coUP & positional & positional \\
bounded parity & PTIME & positional & $d+1$ \\
finitary parity & PTIME & positional & infinite \\
bounded cost-parity & UP $\cap$ coUP & positional & $d+1$ \\
cost-parity & UP $\cap$ coUP & positional & infinite \\
\hline Streett & coNP-complete & exponential & positional \\
bounded Streett & EXPTIME-complete & exponential & exponential \\
finitary Streett & EXPTIME-complete & exponential & infinite \\
bounded cost-Streett & EXPTIME-complete & exponential & exponential \\
cost-Streett & EXPTIME-complete & exponential & infinite \\
\hline
\end{tabular}

Table 1: Overview of computational complexity and memory requirements.

are independent and there is a cost function for every pair of requests and responses. Thus, there are two other possible combinations.

First, consider parity games with multiple cost functions (one for each odd color): a reduction from QBF shows that solving such games is PSPACE-hard. On the other hand, the problem is in EXPTIME, since every such game is a Streett game with costs. Furthermore, one can show that Player 0 needs exponential memory (in the number of odd colors) to implement her winning strategies. All these results even hold for the bounded variant of these game, which is defined as one would expect. In these games, both players need exponential memory. In further research we aim at closing the gap in complexity of solving parity games with multiple cost functions. The second variation are Streett games with a single cost function. Solving finitary Streett games is already EXPTIME-complete and our lower bounds on memory requirements are derived from Streett games. Note that both finitary Streett and classical Streett games can be seen as Streett games with a single cost function. Hence, these games are as hard as Streett games with multiple cost functions.

Finally, there are at least two other directions to extend our results presented here: first, our winning conditions do not cover all acceptance conditions (for automata) discussed in [4, 32. In ongoing research, we investigate whether our techniques are applicable to these more expressive conditions and to winning conditions specified in weak MSO with the unbounding quantifier [3, 5]. Finally, one could add decrement-edges.

\section{REFERENCES}

[1] Roderick Bloem, Krishnendu Chatterjee, Thomas A. Henzinger, and Barbara Jobstmann. Better quality in synthesis through quantitative objectives. In Ahmed Bouajjani and Oded Maler, editors, $C A V$, volume 5643 of LNCS, pages 140-156. Springer, 2009.

[2] Mikołaj Bojańczyk. A bounding quantifier. In Jerzy Marcinkowski and Andrzej Tarlecki, editors, CSL, volume 3210 of LNCS, pages 41-55. Springer, 2004.

[3] Mikołaj Bojańczyk. Weak MSO with the unbounding quantifier. Theory Comput. Syst., 48(3):554-576, 2011.

[4] Mikołaj Bojańczyk and Thomas Colcombet. Bounds in $\omega$-regularity. In LICS [24], pages 285-296.

[5] Mikołaj Bojańczyk and Szymon Toruńczyk. Weak MSO+U over infinite trees. In Christoph Dürr and Thomas Wilke, editors, 29th International Symposium on Theoretical Aspects of Computer Science (STACS 2012), volume 14 of Leibniz International Proceedings in Informatics (LIPIcs), pages 648-660, Dagstuhl, Germany, 2012. Schloss Dagstuhl-Leibniz-Zentrum fuer Informatik. 
[6] Tomás Brázdil, Krishnendu Chatterjee, Antonín Kucera, and Petr Novotný. Efficient controller synthesis for consumption games with multiple resource types. In P. Madhusudan and Sanjit A. Seshia, editors, $C A V$, volume 7358 of $L N C S$, pages 23-38. Springer, 2012.

[7] J. Richard Büchi and Lawrence H. Landweber. Solving sequential conditions by finite-state strategies. Transactions of the American Mathematical Society, 138:pp. 295-311, 1969.

[8] Pavol Černý, Krishnendu Chatterjee, Thomas A. Henzinger, Arjun Radhakrishna, and Rohit Singh. Quantitative synthesis for concurrent programs. In Ganesh Gopalakrishnan and Shaz Qadeer, editors, $C A V$, volume 6806 of $L N C S$, pages 243-259. Springer, 2011.

[9] Krishnendu Chatterjee and Laurent Doyen. Energy parity games. In Samson Abramsky, Cyril Gavoille, Claude Kirchner, Friedhelm Meyer auf der Heide, and Paul G. Spirakis, editors, ICALP (2), volume 6199 of $L N C S$, pages 599-610. Springer, 2010.

[10] Krishnendu Chatterjee and Nathanaël Fijalkow. Infinite-state games with finitary conditions. In Simona Ronchi Della Rocca, editor, CSL, volume 23 of LIPIcs, pages 181-196. Schloss Dagstuhl - LeibnizZentrum fuer Informatik, 2013.

[11] Krishnendu Chatterjee, Thomas A. Henzinger, and Florian Horn. Finitary winning in $\omega$-regular games. ACM Trans. Comput. Log., 11(1), 2009.

[12] Krishnendu Chatterjee, Thomas A. Henzinger, and Florian Horn. The complexity of request-response games. In Adrian Horia Dediu, Shunsuke Inenaga, and Carlos Martín-Vide, editors, LATA, volume 6638 of $L N C S$, pages 227-237. Springer, 2011.

[13] Krishnendu Chatterjee, Thomas A. Henzinger, and Marcin Jurdziński. Mean-payoff parity games. In LICS, pages 178-187. IEEE Computer Society, 2005.

[14] Thomas Colcombet. The theory of stabilisation monoids and regular cost functions. In Susanne Albers, Alberto Marchetti-Spaccamela, Yossi Matias, Sotiris E. Nikoletseas, and Wolfgang Thomas, editors, ICALP (2), volume 5556 of $L N C S$, pages 139-150. Springer, 2009.

[15] Thomas Colcombet and Christof Löding. Regular cost functions over finite trees. In LICS, pages 70-79. IEEE Computer Society, 2010.

[16] Stefan Dziembowski, Marcin Jurdziński, and Igor Walukiewicz. How much memory is needed to win infinite games? In LICS, pages 99-110, 1997.

[17] E. Allen Emerson and Charanjit S. Jutla. Tree automata, mu-calculus and determinacy (extended abstract). In FOCS, pages 368-377. IEEE, 1991.

[18] E. Allen Emerson and Charanjit S. Jutla. The complexity of tree automata and logics of programs. SIAM J. Comput., 29(1):132-158, 1999.

[19] Uli Fahrenberg, Line Juhl, Kim G. Larsen, and Jirí Srba. Energy games in multiweighted automata. In Antonio Cerone and Pekka Pihlajasaari, editors, ICTAC, volume 6916 of LNCS, pages 95-115. Springer, 2011.

[20] Nathanaël Fijalkow and Martin Zimmermann. Cost-Parity and Cost-Streett Games. In Deepak D'Souza, Telikepalli Kavitha, and Jaikumar Radhakrishnan, editors, FSTTCS 2012, volume 18 of LIPIcs, pages 124-135, Dagstuhl, Germany, 2012. Schloss Dagstuhl-Leibniz-Zentrum fuer Informatik.

[21] Kosaburo Hashiguchi. Limitedness theorem on finite automata with distance functions. J. Comput. Syst. Sci., 24(2):233-244, 1982.

[22] Florian Horn, Wolfgang Thomas, and Nico Wallmeier. Optimal strategy synthesis in request-response games. In Sung Deok Cha, Jin-Young Choi, Moonzoo Kim, Insup Lee, and Mahesh Viswanathan, editors, ATVA, volume 5311 of $L N C S$, pages 361-373. Springer, 2008.

[23] Marcin Jurdziński. Deciding the winner in parity games is in UP $\cap$ coUP. Inf. Process. Lett., 68(3):119124, 1998.

[24] 21th IEEE Symposium on Logic in Computer Science (LICS 2006), 12-15 August 2006, Seattle, WA, USA, Proceedings. IEEE Computer Society, 2006.

[25] Donald A. Martin. Borel determinacy. Annals of Mathematics, 102:363-371, 1975.

[26] Robert McNaughton. Playing infinite games in finite time. In Arto Salomaa, Derick Wood, and Sheng Yu, editors, A Half-Century of Automata Theory, pages 73-91. World Scientific, 2000.

[27] Fabio Mogavero, Aniello Murano, and Loredana Sorrentino. On promptness in parity games. In Kenneth L. McMillan, Aart Middeldorp, and Andrei Voronkov, editors, LPAR, volume 8312 of Lecture Notes in Computer Science, pages 601-618. Springer, 2013.

[28] Andrzej Mostowski. Games with forbidden positions. Technical Report 78, University of Gdańsk, 1991. 
[29] David E. Muller and Paul E. Schupp. Simulating alternating tree automata by nondeterministic automata: New results and new proofs of the theorems of Rabin, McNaughton and Safra. Theor. Comput. Sci., 141(1\&2):69-107, 1995.

[30] Daniel Neider, Roman Rabinovich, and Martin Zimmermann. Down the Borel hierarchy: Solving Muller games via safety games. Theoretical Computer Science, 2014. Article in press.

[31] Nir Piterman and Amir Pnueli. Faster solutions of Rabin and Streett games. In LICS [24, pages 275284.

[32] Michael Vanden Boom. Weak cost monadic logic over infinite trees. In Filip Murlak and Piotr Sankowski, editors, MFCS, volume 6907 of LNCS, pages 580-591. Springer, 2011.

[33] Nico Wallmeier, Patrick Hütten, and Wolfgang Thomas. Symbolic synthesis of finite-state controllers for request-response specifications. In Oscar H. Ibarra and Zhe Dang, editors, CIAA, volume 2759 of LNCS, pages 11-22. Springer, 2003.

[34] Martin Zimmermann. Time-optimal winning strategies for poset games. In Sebastian Maneth, editor, CIAA, volume 5642 of $L N C S$, pages 217-226. Springer, 2009. 